\title{
Stellar disks and embedded bars in early-type galaxies
}

\author{
I. 2-D photometric decomposition of 28 southern early-type galaxies \\ C. Scorza ${ }^{1,2, \star}$, R. Bender ${ }^{3, \star}$, C. Winkelmann ${ }^{3}$, M. Capaccioli ${ }^{4}$, and D.F. Macchetto ${ }^{5}$ \\ 1 Landessternwarte, Königstuhl, D-69117 Heidelberg, Germany \\ 2 Centro de Investigaciones de Astronomia CIDA, Apartado 264, Merida 5101-A, Venezuela \\ 3 Universitätssternwarte, Scheinerstr. 1, D-81679 München, Germany \\ ${ }^{4}$ Osservatorio Astronomico Capodimonti, salita Moiariello 16, I-80131 Napoli, Italy and Dipartimento di Scienze Fisiche, \\ Universita' Federico II, I-80100 Napoli, Italy \\ 5 Space Telescope Science Institute, 3700 San Martin Drive, Baltimore, MD 21218, U.S.A.
}

Received December 23, 1997; accepted March 2, 1998

\begin{abstract}
We present photometric disk-bulge decompositions of 28 southern early-type galaxies with types $T<-3$ in either the RC3 or ESO-Lauberts \& Valentijn catalogues. The decomposition method applied here is based on that developed by Scorza \& Bender (1995) but the improved version allows for arbitrary surface brightness profiles of the disk models. We find three types of objects in this sample: bulge-dominated systems, with fully embedded close to edge-on disks; disk-dominated close to edgeon objects and objects with barred disks being modestly inclined down to face-on. Like in Scorza \& Bender (1995), the analysis made here indicates that the superposition of a thin disk and an elliptical bulge can give good account for the morphology of most of the galaxies. We find the disks to have a diversity of surface brightness profiles, the most frequent case being that of a disk with an exponential profile, which becomes steeper at small radii. After disk subtraction, the bulges follow more closely the $r^{1 / 4}$ law. Five of the galaxies show signatures of embedded bar components. These have flat surface brightness profiles at small radii and rectangular shape, which are typical features of barred early-type galaxies. We find that the properties of the galaxies, most notably the disk-to-total ratio, correlates only modestly with the original classification of the galaxies.
\end{abstract}

Send offprint requests to: C. Scorza

* Based on observations obtained at the European Southern Observatory ESO for the ESO Key-project "Towards a physical classification of early-type galaxies". The first two authors are visiting Astronomers at the German-Spanish Astronomical Center, Calar Alto, operated by the Max-Planck-Institut für Astronomie, Heidelberg jointly with the Spanish National Commission for Astronomy.
Key words: galaxies: elliplicals and lenticular — galaxies: structure - galaxies: photometry

\section{Introduction}

The structural similarity between disky ellipticals and S0 galaxies (Carter 1987; Capaccioli 1987; Bender 1988; Nieto 1988; Capaccioli et al. 1990) has been the subject of several photometric and kinematic studies in recent years. The fact that the largest isophotal deviations from perfect ellipses appear in the most elongated ellipticals (Bender et al. 1989a; Capaccioli et al. 1990; Nieto et al. 1991) supports the explanation that pointed isophotes in disky ellipticals are due to the presence of close to edge-on embedded disks. Rix \& White (1990) studied in simulations the detectability of such embedded disks and concluded that disks contributing up to $20 \%$ of the light would remain undetectable in half of all ellipticals because of low inclinations. Kinematic studies demonstrated that, like S0 galaxies, disky ellipticals are in general rapid rotators (Bender 1988; Scorza \& Bender 1995). Further similarities were found to exist in the radio and X-ray properties (Bender et al. 1989a; Eskridge et al. 1995) and in the way that objects populate the Fundamental Plane (Capaccioli et al. 1992).

In order to investigate more quantitatively the disk hypothesis and the family likeness between disky Es and S0 galaxies, it is necessary to study a significant number of objects and analyse their properties in great detail. Work on a few objects has been already done (Capaccioli et al. 1987; Scorza \& Bender 1990; Simien \& Michard 1990; Rix \& White 1990, 1992; Scorza 1992; Cinzano \& 
van der Marel 1994; Iodice et al. 1997). Scorza \& Bender (1995) developed a method which allowed to decompose photometrically 12 disky ellipticals in their disk and bulge components, making possible the direct comparison between the decomposed components of Es and S0 galaxies. They showed that not only the detailed photometric structure of disky ellipticals can be understood in terms of disk+bulge models, but also their detailed kinematic properties. Indeed, the velocity line profiles (VLPs) of disky ellipticals show the same kind of asymmetries found in S0 galaxies (e.g. NGC 3115 in Scorza \& Bender 1995), making the most plausible explanation that of the presence of an embedded disk.

In the present work we apply a similar technique as Scorza \& Bender's (1995) with the aim of analysing a larger sample of early-type galaxies and re-examine the validity of the previous findings. With an improved method we are able to recover disks with arbitrary surface brightness profiles. In a forthcoming paper (Scorza \& Bender 1998) we present a statistical study of disky ellipticals and S0 properties and give insights about the continuity of the Hubble sequence towards smaller $D / B$ ratios.

The present paper is structured as follows. The observations, reduction procedure and isophote analysis are described in Sect. 2. A Morphological characterization of the studied objects is presented in Sect. 3. The decomposition method is introduced in Sect. 4 together with details of the calibration of the images and determination of characteristic parameters. In Sect. 5 we discuss the results of the decomposition, compare our results with previous work and discuss in detail some individual cases. The conclusions and final discussion are drawn in Sect. 6 .

\section{Observations, reduction and isophote analysis}

Twenty-five of the 28 early-type galaxies studied here are part of the ESO key-project sample "Towards a physical classification of early-type galaxies" (Bender et al. 1989b). They were observed at the $1.5 \mathrm{~m}$ Danish and the MPI $2.2 \mathrm{~m}$ telescopes of La Silla, Chile. These galaxies are listed in the RC2 catalogue (de Vaucouleurs et al. 1976) and LV catalogue (Lauberts \& Valentijn 1989) with types $T<-3$ and are brighter than $B_{\mathrm{t}}=13.5 \mathrm{mag}$. The detector was an RCA CCD with a pixel size of $15 \mu \mathrm{m}$, which yielded a resolution of 0.23 arcsec pro pixel. The exposure times were typically of 20 to 30 minutes in the $V$ filter and 7 to 20 minutes in the $R$ filter.

NGC 4473, NGC 4550 and NGC 4564 were observed at the $1.23 \mathrm{~m}$ telescope of the German-Spanish Astronomical center, Calar Alto, Spain. The detector was a GEC CCD with a pixel size of $22 \mu$, which yielded a resolution of 0.46 arsec pro pixel in a field of $3 \times 4$ arcmin.

Along the observations the seeing had in average a value of 2 arcsec. After standard reduction (dark subtraction, flatfield division), the galaxies isophotes were analysed following Bender \& Möllenhoff (1987). This provided the parameters of the best fitting ellipses and the Fouriercoefficients measuring the deviations of the isophotes from ellipses.

The sample was chosen primarily according to the amplitude of the $a_{4} / a * 100$ Fourier coefficient, which has proven to be a very good indicator for the existence of embedded disks (see references above). The sample is a semi-random subset of all objects with positive $a_{4} / a * 100$. However objects with large isophote twists and objects with significant dust absorption were excluded. Table 1 lists the 28 early-type galaxies analysed here, their morphological types and $B_{\mathrm{T}}$ magnitudes according the RC3 catalogue (de Vaucouleurs et al. 1991), the morphological types according to our analysis (Type SB98, see below), the maximum values of the $a_{4} / a * 100$ Fourier coefficient, the filter in which the images were obtained and the galactic standard of rest radial velocities $\left(V_{\mathrm{gsr}}\right)$ in $\mathrm{km} \mathrm{s}^{-1}$ taken from the RC3 catalogue.

Table 1. Basic parameters of the observed galaxies. For each object, we list the classification and $B_{\mathrm{T}}$ according to the RC3, the morphological classification according to our analysis (Type SB98), the maximum values of the $a_{4} / a * 100$ Fourier coefficient, the filter in which the images were taken and the radial velocities corrected to the galactic center $V_{\mathrm{gsr}}$ from the RC3 catalogue

\begin{tabular}{lcccccl}
\hline Object & Type & $B_{\text {T }}$ & Type & $a_{4} / a * 100$ & Filter & $V_{\text {gsr }}$ \\
& RC3 & RC3 & SB97 & & & RC3 \\
\hline E 208-21 & -3.0 & 12.19 & I & 2.5 & $R$ & 812 \\
E 322-60 & -2.2 & 12.10 & II & 2.7 & $R$ & 2401 \\
E 507-21 & -3.0 & 13.23 & II & 1.4 & $R$ & 3008 \\
IC 2552 & -3.0 & 13.42 & III & 4.3 & $R$ & 2884 \\
IC 4329 & -3.0 & 13.42 & I & 1.1 & $V$ & 4402 \\
IC 4889 & -5.0 & 12.06 & I & 1.5 & $V$ & 2473 \\
NGC 1201 & -2.0 & 11.67 & III & 1.9 & $V$ & 1630 \\
NGC 1537 & -2.5 & 11.47 & III & 2.5 & $V$ & 1227 \\
NGC 1700 & -5.0 & 12.20 & Ib & 1.1 & $V$ & 3822 \\
NGC 2271 & -3.0 & 13.17 & I & 1.9 & $R$ & 2408 \\
NGC 2502 & -2.0 & 13.19 & III & 6.0 & $R$ & 867 \\
NGC 2904 & -3.0 & 13.43 & I & 2.3 & $V$ & 2175 \\
NGC 3585 & -5.0 & 10.88 & I & 5.1 & $V$ & 1206 \\
NGC 3706 & -3.0 & 12.38 & I & 1.2 & $V$ & 2776 \\
NGC 3818 & -5.0 & 12.67 & Ib & 2.5 & $V$ & 1364 \\
NGC 4024 & -3.0 & 12.65 & III & 4.0 & $V$ & 1495 \\
NGC 4473 & -5.0 & 11.16 & I & 1.2 & $V$ & 2187 \\
NGC 4550 & -1.5 & 12.56 & II & 2.8 & $V$ & 336 \\
NGC 4564 & -5.0 & 12.05 & II & 2.5 & $V$ & 1066 \\
NGC 4697 & -5.0 & 10.14 & I & 1.9 & $V$ & 1137 \\
NGC 4830 & -2.5 & 13.11 & II & 1.8 & $R$ & 3206 \\
NGC 4831 & -3.0 & 13.39 & II & 1.9 & $V$ & 3180 \\
NGC 5018 & -5.0 & 11.69 & I & 2.0 & $V$ & 2673 \\
NGC 6758 & -4.3 & 12.65 & I & 1.1 & $V$ & 3311 \\
NGC 6851 & -5.0 & 12.69 & I & 1.5 & $V$ & 3023 \\
NGC 7029 & -4.0 & 12.35 & Ib & 3.8 & $V$ & 2754 \\
NGC 7041 & -2.7 & 12.09 & II & 2.6 & $V$ & 1880 \\
NGC 7097 & -5.0 & 12.61 & I & 1.8 & $V$ & 2535 \\
\hline & & & & & &
\end{tabular}

\section{Morphological characterization of objects}

We discrimate between three types of objects in this study depending on morphological appearance. Typical examples of each class are discussed in Sect. 5 and can be found in the Appendix. 
(I) Bulge-dominated objects, i.e. the disks are fully embedded in the bulge and close to edge-on. In this case, the $a_{4} / a$ profile has a well defined peak (where the local diskto-bulge ratio reaches a maximum) and falls to zero at large radii because the disk fades more quickly than the bulge. The rise and fall of the $a_{4} / a$ profile is accompanied by a similar behaviour of the ellipticity, because at both small and large radii, we see the ellipticity of the bulge. We distinguish here another subtype (Ib) which consists in a disk fully embedded in a boxy bulge. In this case, at large radii, the $a_{4} / a$ coefficient becomes negative and the ellipticity continues increasing.

(II) Disk-dominated objects, i.e. the disk dominates in the outer parts, with intermediate to high inclinations. Here, the $a_{4} / a$ profile also falls to zero at large radii because now the disk dominates (instead of the bulge) and the projected disk has pure elliptical isophotes. There are two indicators which allow to separate this case from case (I): (a) the ellipticity profile and (b) the surface brightness profile. If the disk becomes dominant at large radii, then the ellipticity remains constant in radius and directly reflects the inclination angle of the disk. Furthermore the surface brightness profile is generally closer to an exponential than to an $r^{1 / 4}$ profile.

(III) Objects with barred disks being modestly inclined down to face-on. These objects are characterized by correlated and rather abrupt changes in diskiness $\left(a_{4} / a\right)$, position angle and ellipticity. Such behaviour is also observed in barred S0s (Magrelli et al. 1992; Shaw et al. 1993), which suggests that the isophotal twists in ellipticals could be caused by embedded bars (Nieto et al. 1992). Barred objects close to edge-on can generally not be distinguished from cases (I) or (II).

These three cases require different treatments. Case (I) and (II) will be decomposed by variants of the disk-bulge decomposition method developed by Scorza \& Bender (1995). Case (III) cannot be decomposed reliably but the bar hypothesis can be tested via subtraction of a bulge model.

\section{Photometric decomposition: The method}

\subsection{Decomposition of close to edge-on systems}

In this case, the analysis procedure follows two modes, depending on whether the disk is completely embedded in the bulge or whether it is dominant in the outer parts. As already mentioned, the decomposition method applied here is a modified version of the method described in Scorza \& Bender (1995). In the latter work it was found that the modeling of twelve early-type galaxies required disk models with profiles departing from the exponential behaviour. These were constructed by adding several exponential functions, most frequently two exponentials were required. In the present work we have developed an automatized method to determine the optimal flux intensities of the disk models without constraints on the shapes of the disk profiles. As a first step, the initial values of an exponential disk model were guessed as described in Scorza \& Bender (1995). The models are described by a central surface brightness $S B_{0}$, a scale length $r_{\mathrm{d}}$ and an inclination angle $i$. After subtraction of this input disk model (convolved to the appropriate seeing) from the galaxy image, an isophote analysis of the remaining bulge was carried out. This analysis yielded, among several other parameters, the $a_{4} / a$ amplitudes along the major axis. The radial flux of the input disk model was then corrected according to the amplitude of the remaining $a_{4} / a$ signature of the bulge via:

$F_{\mathrm{b}}=F_{\mathrm{a}}(r) \cdot\left(1.0+a_{4} / a(r) / \mathrm{const}\right)$

where $F_{\mathrm{a}}$ is the initial flux, $F_{\mathrm{b}}$ the corrected one and $r$ the radius. With this corrected flux profile, a new disk model was constructed and subtracted from the galaxy image. The procedure was repeated until the isophotes of the bulge show $a_{4} / a \sim 0$. If it was found that the iteration did fail to converge properly, an input disk model with either a higher or a lower surface brightness was used.

Simulations of the decomposition procedure with perfect galaxy models showed that both input disks with too high or too low surface brightness did converge to the true disk. However, the convergence from brighter input disk models was in general better than from fainter input disks.

In the present version of the method the surface brightness profile of the disk is left free and can take any shape, provided that after disk subtraction the remaining bulge shows elliptical isophotes. The method described so far gave satisfactory results for objects with embedded disks (case I in Sect. 3). In case of a boxy bulge (case I.b) the disk profile was modified in such way that after disk subtraction, the remaining bulge showed also boxy isophotes and therefore negative $a_{4} / 4$ values (e.g. see the case of NGC 3818, Fig. A15 in the Appendix).

If the disk was found to dominate at large radii (case II), the iteration procedure was similar, however, the initial disk parameters had to be estimated more carefully. This was done in two steps. First, a disk model was constructed following the above procedure. This model failed to produce a convincing solution in the outer disk dominated parts (specially the surface brightness profile) but allowed to estimate the bulge and disk profile in the inner parts, where the bulge still dominates. The bulge profile was then fitted by an $r^{1 / 4}$ model and extrapolated out to radii where the disk dominates. The such constructed bulge model was subtracted from the galaxy and the remaining disk was used as input disk for a further iteration. In this way, a satisfactory decomposition was achieved.

Figure 1 illustrates this procedure for NGC 4564. In Fig. 1a we show the surface brightness profile (uppermost panel) and the $a_{4}$ and ellipticity profiles (middle and lower panel) before (filled circles) and after (crosses) subtraction 


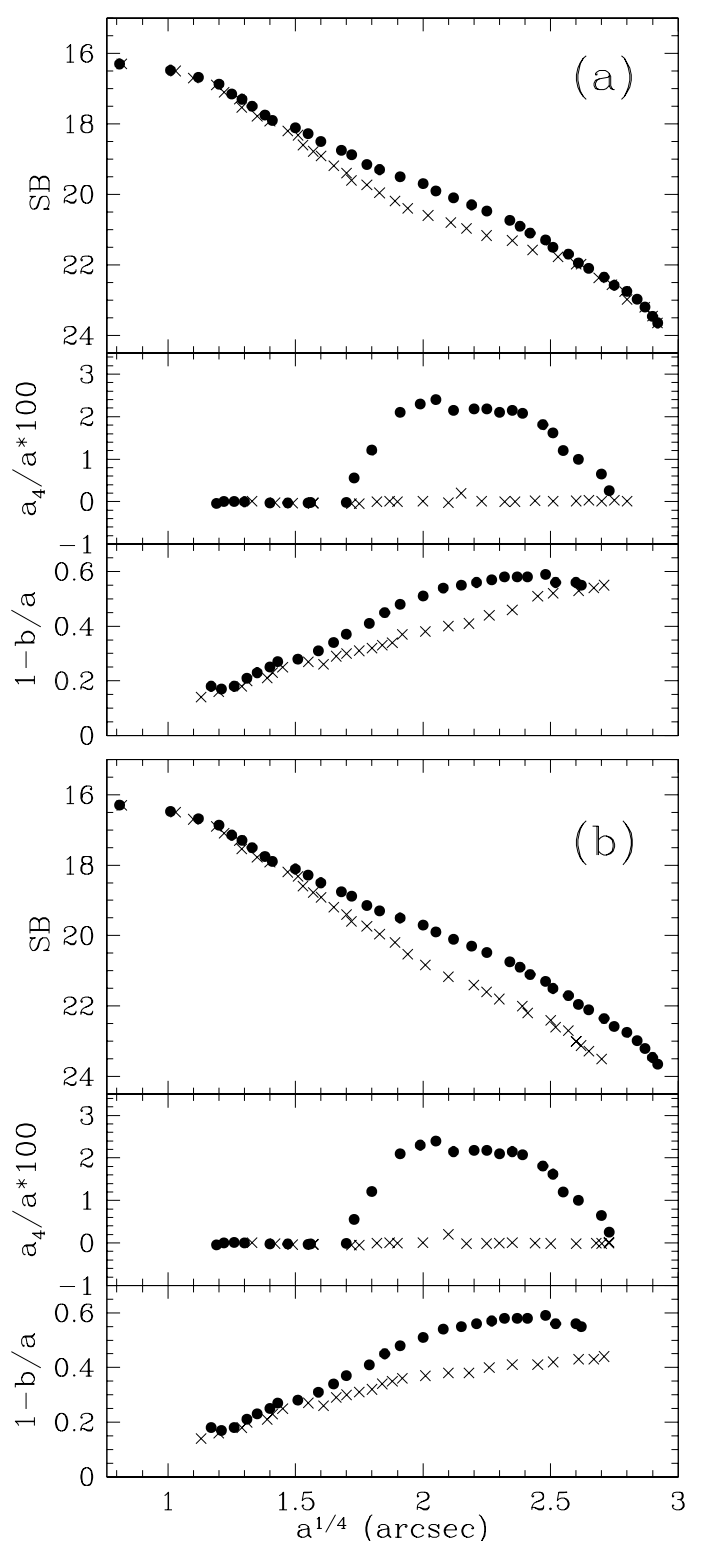

Fig. 1. Surface brightness, $a_{4} / a * 100$ and ellipticity profiles of NGC 4564 a) before (filled circles) and after (crosses) the subtraction of a too small disk and $\mathbf{b}$ ) before and after subtraction of a brighter and larger disk (see text)

of a first (too small) disk model. Although the subtraction of this disk model yields a bulge with vanishing $a_{4} / a$, the brightness profile of the bulge still shows signatures of the disk: mainly a hump at radii $>23 \operatorname{arcsec}\left(2.5\right.$ in the $a^{1 / 4}$ scale) and ellipticity values which continue increasing at large radii. After having modified the bulge profile and having constructed and subtracted an $r^{1 / 4}$ bulge model, a second input disk was obtained. With the latter, a better solution (shown in Fig. 1b) was achieved. The change in the ellipticity profile is noticeable (lower panel in Fig. 1b): the bulge has lower ellipticity when compared with the previous solution.

\subsection{Decomposition of close to face-on barred-systems}

As already mentioned in Sect. 3, a detailed decomposition of these systems is not possible by means of the present method. However, the bar hypothesis infered from the behaviour of the Fourier coefficients can be tested after subtraction of a bulge model and examination of the residual structures. We follow here the same procedure applied in Scorza \& Bender (1995) to recover the embedded bar in NGC 4660. As a first step, the decomposition method described above was applied. This yielded an approximated bulge profile from which a bulge model having constant position angle and ellipticity was constructed. This bulge model was then subtracted from the galaxy image and the residual structures were analysed. Notice that in this case it is assumed that the observed twists and abrupt ellipticity changes are due to the embedded bar component and therefore the constructed bulge-model is free from these features. Although triaxiality can also lead to projected twists of the isophotes (see e.g. Benacchio \& Galleta 1980) the bar hypothesis is more likely for isotropic oblate rotators, which is the case of the galaxies examined here. In all galaxies showing the typical behaviour of the Fourier coefficients described in Sect. 3 (case III), elongated barredlike structures could be recovered after the bulge-model subtraction. Examples are shown in Sect. 5.2.3.

\subsection{Calibration and determination of characteristic parameters}

Similar to Scorza \& Bender (1995), the zero point calibration was done here by comparing integrated fluxes within apertures with values given in the literature (Poulain 1993). After calibration, we derived characteristic parameters from growth-curve-fitting: the half light radius $r_{\mathrm{e}}$, the surface brightness $S B_{\mathrm{e}}$ at $r_{\mathrm{e}}$ and the total apparent magnitude $m_{\text {tot }}$. All these parameters were derived for galaxies, bulges and disks separately. For the latter, both $r_{\mathrm{e}}$ and $S B_{\mathrm{e}}$ were corrected to face-on.

Because the disks analysed here often have surface brightness profiles which deviate from exponentials and central surface brightnesses are ill-defined (see Scorza \& Bender 1995), the best way to characterize the disks is then by their mean effective surface brightness $\langle S B\rangle_{\mathrm{e}}$ :

$\langle S B\rangle_{\mathrm{e}}=m_{\text {tot }}+5 \cdot \log \left(r_{\mathrm{e}}\right)+1.995$.

However, given that throughout the literature the disks of late-type galaxies are parameterized by the extrapolated central surface brightness $S B_{0}$ and scale length $r_{\mathrm{d}}$, we have determined also for comparison purposes the $S B_{0}$ for the disks and a mean $r_{\mathrm{d}}$ value obtained from the fit of exponential growth curves to the disks (for details see Scorza $\&$ Bender 1995). In this case, the mean $r_{\mathrm{d}}$ is representative of the dominant exponential part of the disk profiles. The $S B_{0}$ values were obtained directly from the central intensities of the disk models and corrected to face-on. Due to 
systematic effects, assumptions concerning bulge shapes, non-exponentiality of the disks and seeing, the errors for these parameters could not be estimated reliably for each case. In general, however, from exploring a range of models we have estimated the following rough errors: $\Delta S B_{0}$ $\simeq 0.2$ and $\Delta r_{\mathrm{d}} \simeq 20 \%$ to $30 \%$.

The distances to the galaxies were determined from the radial velocities given in the literature (RC3 catalogue) and a Hubble constant of $H_{0}=75 \mathrm{~km} \mathrm{~s}^{-1} \mathrm{Mpc}^{-1}$ ). With these quantities, the absolute magnitudes and scale lengths of the disks in kpc were derived. The absolute magnitudes and the $\langle S B\rangle_{\mathrm{e}}$ were corrected for dust absorption in the galactic plane according to Burstein (1984). The magnitudes and surface brightness of the galaxy images obtained only in the $R$ band were transformed to the $V$ band following Peletier (1989):

$V=R-(B-V)+\frac{(B-V)+0.013}{0.620}$

where the $(B-V)$ were taken from the RC3 catalogue.

\section{Results}

In this section we discuss the results of the photometric decomposition, compare our results with previous work and present details on individual cases. The surface brightness profiles of the galaxies, their bulge and disk components are shown in the appendix A together with the ellipticity, position angle and Fourier coefficient profiles prior and after disk subtraction. The determined disk and bulge parameters are listed in Table 2 together with the estimated disk-to-bulge ratios $D / B$ and other relevant parameters.

\subsection{Comparison with previous work}

We have compared the results obtained here for three galaxies (NGC 3585, NGC 4697 and IC 2552) with those given in previous work by Scorza \& Bender (1995) and Scorza (1993), and in the case of NGC 3585, also with the recent decomposition made by Michard (1997). The comparison enables us to test the automatized version of the method applied here, and particularly the assumption on the shape of the disk profiles, which in Scorza \& Bender (1995) were taken to be the sum of two or more exponentials, and here left free.

The surface brightness profiles of the comparison galaxies, their bulges and disks are shown in Figs. 2a, 2b and 2c. Shown are the bulge and disk surface brightness as determined here (full line) and by Scorza \& Bender (1995) (dashed line). In the case of NGC 3585 (Fig. 2a), the dotted line shows the decomposition of Michard (1997). The differences in the surface brightness profiles of the bulges at large radii are probably caused by differences in the determination of the sky background in the three mentioned works. This seems to be indeed the case since

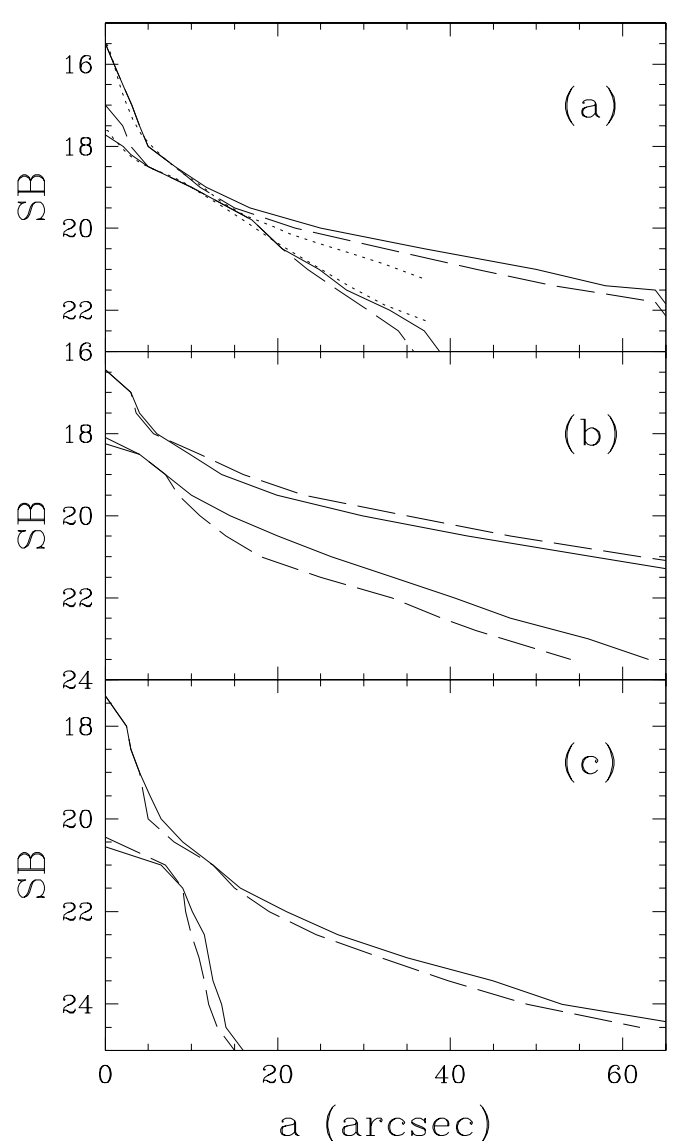

Fig. 2. Comparison with previous work. Surface brightness profiles $S B$ (in mag/ $/ \operatorname{arcsc}^{2}$ ) of the bulge and disk components of: a) NGC 3585 as determined here (full line), by Scorza \& Bender (1995) (dashed lines) and by Michard (1997) (dotted line); b) NGC 4697 as determined here (full line) and by Scorza \& Bender (1995) (dashed lines), and c) IC 2552 as determined here (full line) and in Scorza (1993) (dashed lines)

the profiles in all works lay very close to each other at small radii, but diverge at large radii (in a more extremely way Michard's). The same holds for the other comparisons shown in Figs. 2b and 2c.

The surface brightness profiles of the disks show at small radii $(<3$ arcsec) significant differences which are most probably due to the uncertainties caused by seeing. Outside these radii the profiles have a similar run in all decompositions, but at larger radii the difference becomes again noticeable. The disk profiles derived here are slightly brighter at large radii than those derived by Scorza \& Bender (1995). This can in part be due to the non-exponentiality of the disk profiles.

Another difference resides in the higher central surface brightness values of the latter work, explained by the fact that the authors combined two exponentials to construct their models. Thus, the smaller scale lengths and higher central surface brightnesses seem to be due to a systematic difference between the two decomposition techniques and 
Table 2. Derived parameters of the bulge and disk components. For each object, we list the disk-to-bulge ratio $D / B$, the total magnitude $V_{\mathrm{Tg}}$, effective surface brightness $S B_{\mathrm{eg}}$ and effective radius $r_{\mathrm{eg}}$ of the galaxy; the total magnitude $V_{\mathrm{Tb}}$, the effective surface brightness $S B_{\text {eb }}$ and effective radius $r_{\text {eb }}$ of the bulge component; the total magnitude $V_{\mathrm{Td}}$, effective surface brightness $S B_{\text {ed }}$ and effective radius $r_{\text {de }}$ of the disk component; the inclination $i$, and the face-on corrected central surface brightness $S B_{0}$ and mean scale length of the disks $r_{\mathrm{d}}$. The disk parameters were corrected to face-on. The radii are all given in kpc. Typical errors are $\Delta S B_{0} \simeq 0.2$ and $\Delta r_{\mathrm{d}} \simeq 20 \%-30 \%$

\begin{tabular}{|c|c|c|c|c|c|c|c|c|c|c|c|c|c|}
\hline Object & $D / B$ & $V_{\mathrm{Tg}}$ & $S B_{\text {eg }}$ & $r_{\mathrm{eg}}$ & $V_{\mathrm{Tb}}$ & $S B_{\mathrm{eb}}$ & $r_{\mathrm{eb}}$ & $V_{\mathrm{Td}}$ & $S B_{\text {ed }}$ & $r_{\text {ed }}$ & $i$ & $S B_{0}$ & $r_{\mathrm{d}}$ \\
\hline E $208-21$ & 0.09 & 11.32 & 19.37 & 1.05 & 11.43 & 20.11 & 1.46 & 13.89 & 19.63 & 0.36 & 72 & 18.88 & 0.21 \\
\hline E $322-60$ & 0.44 & 12.19 & 19.37 & 2.00 & 12.60 & 18.40 & 0.91 & 13.45 & 22.31 & 4.32 & 73 & 21.04 & 2.57 \\
\hline E $507-21$ & 0.20 & 12.36 & 20.15 & 3.12 & 12.56 & 21.27 & 5.04 & 14.31 & 22.06 & 3.06 & 70 & 21.56 & 1.82 \\
\hline IC 2552 & 0.02 & 11.75 & 21.52 & 7.80 & 11.78 & 21.81 & 8.99 & 15.85 & 21.77 & 1.33 & 72 & 22.45 & 0.79 \\
\hline IC 4329 & 0.01 & 10.46 & 20.94 & 14.9 & 10.47 & 23.54 & 50.7 & 15.88 & 23.04 & 3.22 & 70 & 21.46 & 1.91 \\
\hline IC 4889 & 0.06 & 11.02 & 19.87 & 4.00 & 11.09 & 20.45 & 5.27 & 14.03 & 20.24 & 1.19 & 63 & 19.56 & 0.70 \\
\hline NGC 1201 & 0.08 & 10.42 & 20.68 & 4.73 & 10.51 & 21.25 & 6.28 & 13.10 & 23.34 & 4.70 & 68 & 19.82 & 2.80 \\
\hline NGC 1537 & 0.06 & 10.76 & 19.44 & 1.80 & 10.70 & 20.52 & 3.20 & 13.69 & 21.32 & 1.06 & 71 & 21.12 & 0.63 \\
\hline NGC 1700 & 0.03 & 11.22 & 19.67 & 5.03 & 11.37 & 20.98 & 9.43 & 13.44 & 19.59 & 1.75 & 57 & 18.66 & 1.04 \\
\hline NGC 2271 & 0.20 & 12.01 & 19.57 & 2.87 & 12.21 & 20.44 & 4.15 & 13.95 & 20.95 & 2.22 & 67 & 20.11 & 1.32 \\
\hline NGC 2502 & 0.12 & 12.71 & 20.2 & 1.00 & 12.84 & 20.05 & 0.87 & 15.09 & 25.03 & 3.09 & 80 & 26.10 & 1.84 \\
\hline NGC 2904 & 0.19 & 12.47 & 19.83 & 2.13 & 12.66 & 20.88 & 3.38 & 14.46 & 21.26 & 1.64 & 66 & 20.00 & 0.97 \\
\hline NGC 3585 & 0.08 & 9.67 & 19.87 & 3.75 & 9.76 & 21.23 & 7.89 & 12.42 & 20.65 & 1.52 & 77 & 19.32 & 0.90 \\
\hline NGC 3706 & 0.06 & 10.98 & 20.34 & 6.04 & 11.05 & 20.74 & 7.16 & 14.06 & 21.29 & 2.27 & 67 & 20.12 & 1.35 \\
\hline NGC 3818 & 0.12 & 11.50 & 20.91 & 2.78 & 11.62 & 23.49 & 11.3 & 13.93 & 20.39 & 0.72 & 64 & 19.60 & 0.42 \\
\hline NGC 4024 & 0.04 & 11.53 & 20.57 & 2.48 & 11.58 & 21.31 & 3.65 & 14.99 & 22.37 & 1.16 & 68 & 21.67 & 0.69 \\
\hline NGC 4473 & 0.21 & 10.28 & 19.07 & 3.29 & 10.50 & 19.89 & 4.44 & 12.13 & 19.72 & 1.89 & 65 & 17.64 & 1.12 \\
\hline NGC 4550 & 0.21 & 11.42 & 19.78 & 0.43 & 11.64 & 20.81 & 0.66 & 13.27 & 21.63 & 0.43 & 77 & 20.32 & 0.25 \\
\hline NGC 4564 & 0.40 & 10.95 & 19.56 & 1.47 & 11.32 & 19.44 & 1.06 & 12.30 & 21.82 & 2.24 & 73 & 20.84 & 1.33 \\
\hline NGC 4697 & 0.07 & 9.26 & 20.41 & 5.05 & 9.34 & 21.15 & 7.36 & 12.17 & 20.97 & 1.72 & 70 & 19.51 & 1.02 \\
\hline NGC 4830 & 0.08 & 12.59 & 20.96 & 4.17 & 12.69 & 21.53 & 5.50 & 15.27 & 22.41 & 2.37 & 70 & 19.96 & 1.41 \\
\hline NGC 4831 & 1.04 & 12.88 & 21.63 & 5.28 & 13.65 & 20.98 & 2.76 & 13.62 & 24.46 & 13.9 & 65 & 20.74 & 8.2 \\
\hline NGC 5018 & 0.11 & 10.73 & 19.41 & 4.05 & 10.85 & 19.94 & 5.02 & 13.19 & 21.15 & 2.91 & 66 & 19.48 & 1.73 \\
\hline NGC 6758 & 0.06 & 11.43 & 20.37 & 5.63 & 11.50 & 20.66 & 6.30 & 14.46 & 22.41 & 3.57 & 60 & 20.60 & 2.12 \\
\hline NGC 6851 & 0.07 & 11.73 & 19.55 & 3.03 & 11.81 & 19.88 & 3.46 & 14.65 & 21.52 & 1.95 & 66 & 20.48 & 1.16 \\
\hline NGC 7029 & 0.05 & 11.30 & 20.41 & 4.71 & 11.35 & 21.04 & 6.50 & 14.60 & 22.10 & 2.25 & 73 & 21.49 & 1.34 \\
\hline NGC 7041 & 0.88 & 10.97 & 20.34 & 3.71 & 11.66 & 20.65 & 2.86 & 11.79 & 22.16 & 5.87 & 73 & 18.94 & 3.49 \\
\hline NGC 7097 & 0.09 & 11.59 & 19.93 & 3.06 & 11.70 & 20.47 & 3.79 & 14.15 & 21.50 & 1.93 & 65 & 20.24 & 1.15 \\
\hline
\end{tabular}

not to an error in the determination of the disk parameters. The same is true for the slightly higher apparent magnitudes of the disks derived in this work. However, the influence of the different shapes of the disk profiles as found here and in Scorza \& Bender (1995) on the $D / B$ ratio is in comparison quite small. The values found here are $\sim 2 \%$ higher. It is worthy to mention that the inclination values found with both methods agree perfectly. The difference is smaller than $3^{\circ}$.

The luminosity profile of the disk in NGC 4550 determined here, agrees well in the range $r=10-20$ arcsec with the profile derived by Rix et al. (1992) from the kinematic decomposition of the VLPs. As mentioned in the introduction, the asymmetric VLPs observed in disky ellipticals make an embedded disk component the most plausible explanation for the peakness of the isophotes.

\subsection{Discussion of individual cases}

We turn now to discuss in detail the decomposition of six galaxies belonging to the three types of objects described in Sect. 3.

\subsubsection{Bulge-dominated objects}

Several clean examples for this type of objects have been discussed in Scorza \& Bender (1995). We present here further details for ESO $208-21$ and NGC 3818.

ESO 208-21: The disk of this galaxy is fully embedded in the bulge component, as indicated by both the $a_{4}$ Fourier coefficient and ellipticity profiles (see Fig. A1 in the Appendix). These show single maxima at intermediate radii and decline at large radii, where the bulge dominates. The surface brightness profile of the disk was found to be exponential over a large radius range and to turn slightly steeper inside 10 arcsec. The disk is very compact, with a mean effective surface brightness of $19.6 \mathrm{mag} / \mathrm{arcsec}^{2}$ and an effective radius of $0.36 \mathrm{kpc}$. 
NGC 3818: This object is representative of the class having a fully embedded disk in a boxy bulge. The $a_{4}$ profile of the original galaxy has negative values at large radii (see Fig. A15 in the Appendix). The disk has been removed in a way to give the bulge constant boxiness. Uncertainties associated with this procedure, e.g. on the $D / B$ ratio, are discussed in Scorza \& Bender (1995). The disk profile is exponential all throughout.

\subsubsection{Disk-dominated objects}

NGC 4831: The $a_{4}$ Fourier coefficient of this galaxy shows a single maximum at 16 arcsec (see Fig. A22 in the Appendix) and beyond this radius decays while the ellipticity continues rising. Like NGC 4564 (described in detail in Sect. 4.1) two steps were applied in the decomposition of this object. The solution with the brighter disk yielded a $D / B=1.04$. The disk profile of this galaxy is exponential at large radii and turns steeper at small radii. The disk was found to have an inclination of $65^{\circ}$ degrees.

NGC 7041: This is also an object with a dominant disk, as indicated by the high $a_{4}$ and ellipticity values. The disk has a profile well represented by a power+exponential law (see Fig. A27 in the Appendix). The decomposition made here agrees with the classification of the RSA (S0) and RC3 catalogue $(T=-2.7)$.

\subsubsection{Barred objects}

IC 2552: This object shows the correlated behaviour of the $a_{4}$, position angle and ellipticity described in Sect. 4.1 (case III). After the bulge-model subtraction, an elongated bar-like structure is observed. At the radius where this sub-structure becomes dominant, the ellipticity, $a_{4}$ and position angle increase abruptly, and fall again at radii where the sub-structure fades. Outside this radius the galaxy turns very round (ellipticity $\sim 0.04$ ). The twist observed in this galaxy is $30^{\circ}$. The surface brightness profile of the sub-component is exponential at large radii and flattens in the inner parts (see Fig. A4 in the appendix). This suggests the presence of a face-on disk with a bar. Figure $3 \mathrm{a}$ shows the residual bar-like structure which extends 8 arcsec from the center.

NGC 4024: This objects shows the same characteristics as IC 2552 (see Fig. A16 in the Appendix). After the bulgemodel subtraction, the bar-like structure shown in Fig. $3 \mathrm{~b}$ becomes visible.

\section{Conclusions and discussion}

In this work we have presented photometric data of 28 early-type galaxies with classification $T<-3$ in either

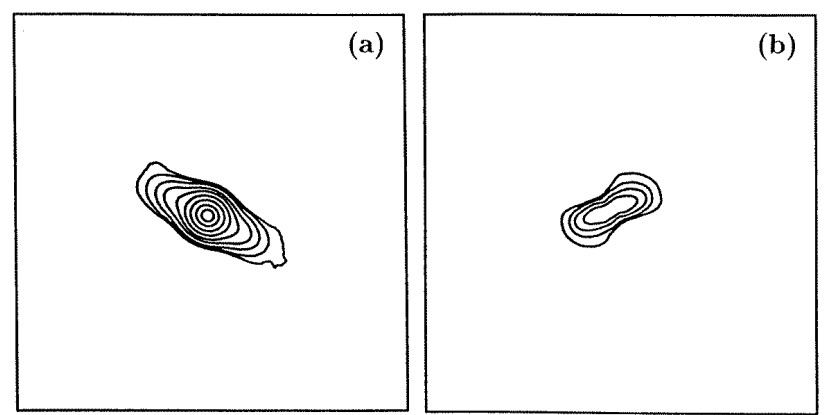

Fig. 3. Residual bar-like structures in a) IC 2552, showing outer rectangular and inner peaked isophotes (the scale is $20 \times 20$ arcsec), and b) in NGC 4024, showing rectangular isophotes. The scale here is $22 \times 22 \operatorname{arcsec}$

the RC3 or ESO-Lauberts \& Valentijn catalogues and their decompositions into bulge and disk. The decomposition method applied here is based on that developed by Scorza \& Bender (1995) but the improved method allows for arbitrary surface brightness profiles of the disk models. We find three types of objects in this sample: bulge-dominated, with fully embedded close to edge-on disks; close to edge-on disk-dominated objects and objects with barred disks being modestly inclined down to faceon. Naturally we expect among the edge-on objects also barred ones but these cannot be detected easily because of the lack of unambiguous features. Bars are also difficult to identify in cases where the bar major axis is aligned with the projected major axis of the disk component. Due to the high flattening of the bar component, the galaxy may be interpreted as being more inclined than it actually is. The described ambiguities can be critical when interpreting the distribution of inclination angles for a sample of objects. The tendency is that one will always find an apparent overabundance of more edge-on objects relative to more face-on ones. The fact that face-on barless disks can hardly be identified at all if the disk-to-bulge ratio is lower than $30 \%$, worsens this problem further.

Like in Scorza \& Bender (1995), the analysis made here indicates that the superposition of a thin disk and an elliptical bulge can give good account for the morphology of most of the early-type galaxies selected here. We find that the disks have a diversity of surface brightness profiles, the most frequent case being that of a disk with an exponential profile, which becomes steeper at small radii. We notice that after disk subtraction the bulge profiles follow more closely an $r^{1 / 4}$ law and many of them show still increasing ellipticity profiles. Five of the galaxies show signatures for embedded bar components. These become visible once the bulge has been subtracted. In three of these objects the surface brightness profile of the bar flattens in the inner parts. It is noticeable that this kind of profiles have been observed in many early-type SB0 galaxies (Elmegreen \& Elmegreen 1985) and that their 
isophotal shape is rectangular, also typical of barred earlytype galaxies (Athanassoula et al. 1990).

We find that the properties of the galaxies, most notably the disk-to-bulge ratio, correlate only modestly with the original classification of the galaxies. An extreme case is NGC 4564 , classified as $T=-5$ (E0) in the RC3 catalogue and which has one of the largest $D / B$ ratio of the examined sample $(D / B=0.40)$. Other examples for large diskiness are NGC 1700, NGC 3818, NGC 4473 and NGC 5018.

In a forthcoming paper (Scorza \& Bender 1998) we present a statistical analysis of the sample of galaxies examined here and address questions concerning the continuity of the Hubble sequence towards smaller $D / B$ ratios and the family likeness between disky ellipticals and S0s.

\section{Appendix A}

The results of the photometric decomposition of the 28 galaxies are shown in this appendix. The figures follow the same order of the list of objects in Table 1. The upper left panel shows the surface brightness profiles of each galaxy along the major and minor axes (filled squares), the same profiles of the bulge (crosses) and the disk profile along the major axis (full line), all plotted vs. $a^{1 / 4}$. The same profiles plotted vs. the semi-major axis $a$ are shown in the upper right panel. In the left lower panels are shown: the ellipticity $1-b / a, a_{3} / a \cdot 100, a_{4} / a \cdot 100$, and $a_{6} / a \cdot 100$ profiles before (filled squares) and after (crosses) disk subtraction. At the right lower panels: the position angle PA, $b_{3} / a \cdot 100 b_{4} / a \cdot 100$ and $b_{6} / a \cdot 100$ before (filled squares) and after (crosses) disk subtraction.

Acknowledgements. We wish to honour the memory of our dear friend and coinvestigator in this project Dr. Jean-Luc Nieto whose enthusiasm and scientific curiosity we still miss. CS thanks R. Michard for his unpublished data on NGC 3585. This work was supported by the DFG (Sonderforschungsbereich SFB 328 and SFB 375).

\section{References}

Athanassoula E., Morin S., Wozniak H., Puy D., Pierce M.J., Lombard J., Bosma A., 1990, MNRAS 245, 130

Benacchio L., Galleta G., MNRAS 193, 880

Bender R., Möllenhoff C., 1987, A\&A 177, 71
Bender R., 1988, A\&A 193, L7

Bender R., Surma P., Döbereiner S., Möllenhoff C., Madejsky R., 1989a, A\&A 217, 35

Bender R., Capaccioli M., Macchetto D., Nieto J-L., 1989b, ESO Messenger 55, 6

Capaccioli M., 1987, in IAU Symposium 127, Structure and Dynamics of Elliptical Galaxies, de Zeeuw T.P. (ed.). Dordrecht: Reidel, p. 47

Capaccioli M., Held. E.V., Nieto J.-L., 1987, AJ 94, 1519

Capaccioli M., Caon N., Rampazzo R., 1990, MNRAS 242, 24 Carlberg R.G., 1986, ApJ 310, 593

Capaccioli M., Caon N., D’Onofrio M., 1992, MNRAS 259, 323

Carter, 1987, ApJ 312, 514

Cinzano P., van der Marel R.P., 1994, MNRAS 270, 32

Eskridge P., Fabbiano G., Kim D., 1995, ApJ 442, 543

Elmegreen B.G., Elmegreen D.M., ApJ 288, 438

Iodice E., D'Onofrio M., Capaccioli M., 1997, in the Second Stromlo Symposium, The Nature of Elliptical Galaxies, Arnaboldi M., Da Costa G.S. (eds.)

Lauberts A., Valentijn E.A, 1989, The Surface Photometric Catalogue of the ESO-Upsala Galaxies Catalogue. ESO, Garching

Magrelli G., Bettoni D., Galleta G., 1992, MNRAS 256, 500

Michard R., 1997, A\&A (in press)

Nieto J-L., 1988, $2^{\text {da }}$ Reunion de Astronomia Extragalactica, Academia Nacional de Ciencias de Cordoba, Cordoba Argentina, p. 239

Nieto J.-L., Poulain P., Davoust E., Rosenblatt P., 1991, A\&AS 88,559

Nieto J.-L., Bender R., Poulain P., Surma, P., 1992, A\&A 257, 97

Poulain P., 1993, A\&AS 103, 315

Rix H.-W., White S., 1990, ApJ 362, 52

Rix H.-W., White S., 1992, MNRAS 254, 389

Rix H-W., Franx M., Fisher D., Illingworth G., 1992, ApJ 400, L5-L8

Scorza C., Bender R., 1990, A\&A 235, 49

Scorza C., 1992, in ESO/EIPC-workshop on Structure, Dynamics and Chemical Evolution of Early-type Galaxies, Danzinger J. (ed.)

Scorza C., 1993, Ph.D thesis, Heidelberg-University

Scorza C., Bender R., 1995, A\&A 293, 20

Shaw M.A., Combes F., Axon D.J., Wright G.S., 1993, A\&A 273,31

Simien F., Michard R., 1990, A\&A 227, 11

de Vaucouleurs G., de Vaucouleurs A., Corwin H., 1976, Second Reference Catalogue of Bright Galaxies. Austin, University of Texas Press

de Vaucouleurs G., de Vaucouleurs A., Corwin H., 1991, Third Reference Catalogue of Bright Galaxies. Springer Verlag 


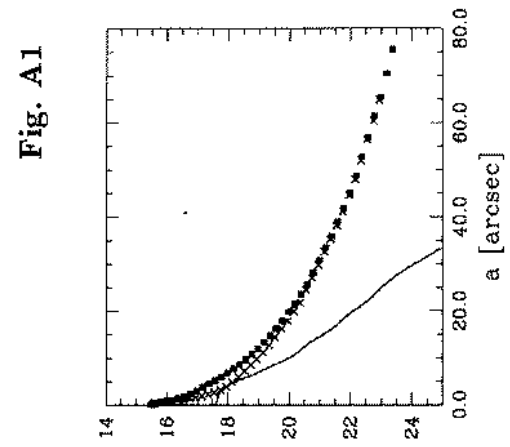

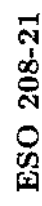

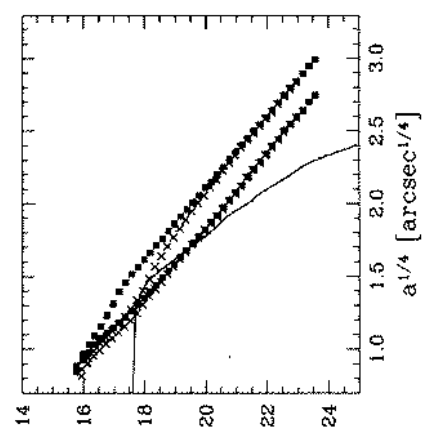

[zosole seux] dS

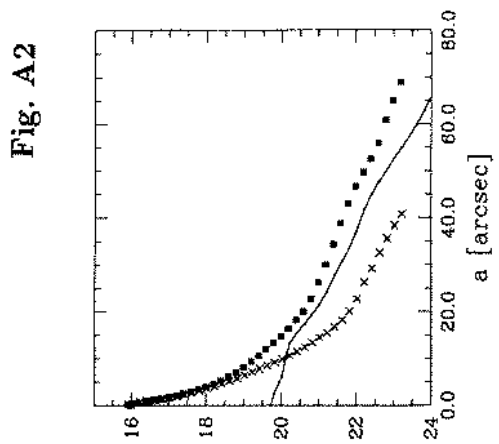

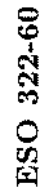

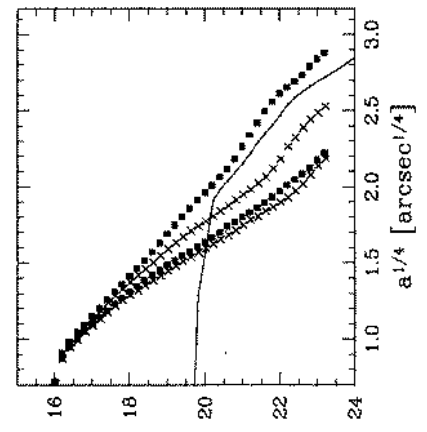

$[z-30 \operatorname{cose}$ sew] as

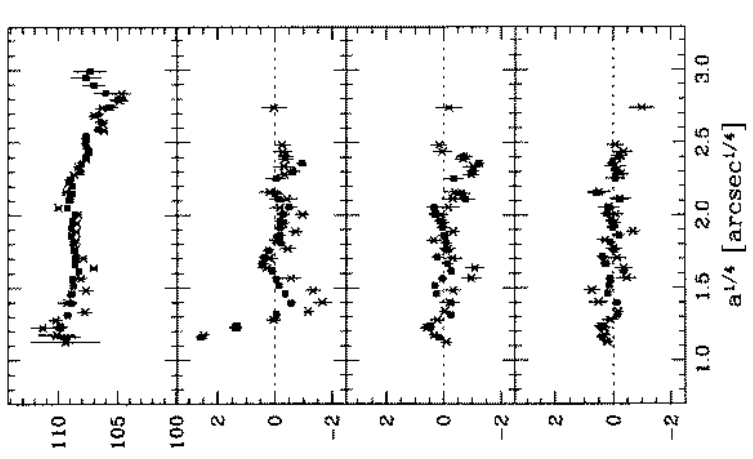

$\forall \mathrm{d} \quad 001 * \mathrm{e} / \mathrm{k} \mathrm{q} \quad 001 * \mathrm{e} /{ }^{*} \mathrm{q} \quad 001 *^{\mathrm{e}} /{ }^{\circ} \mathrm{q}$

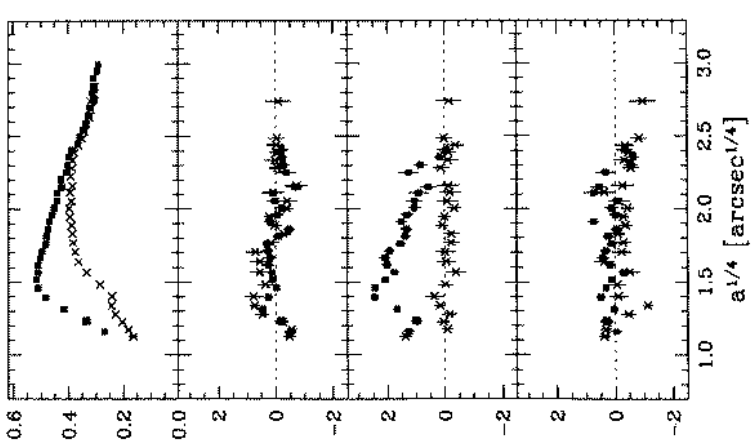

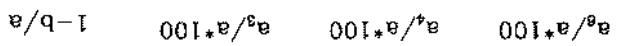

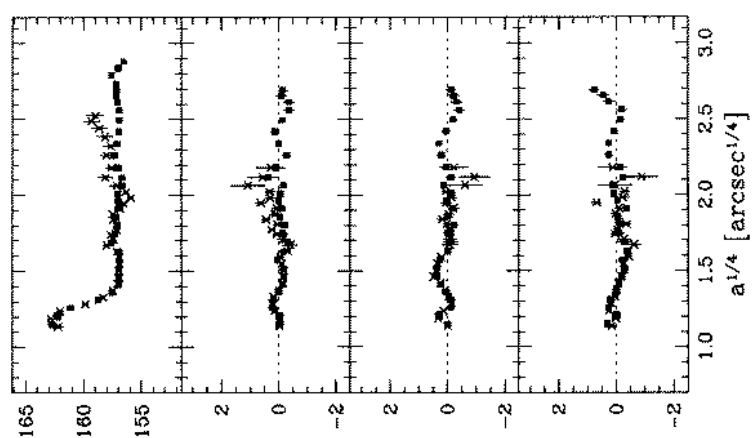

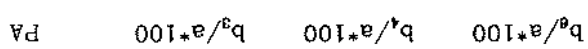

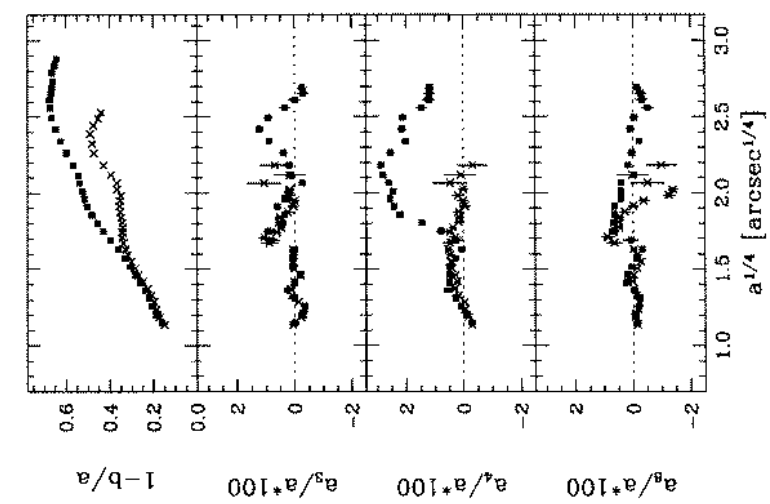




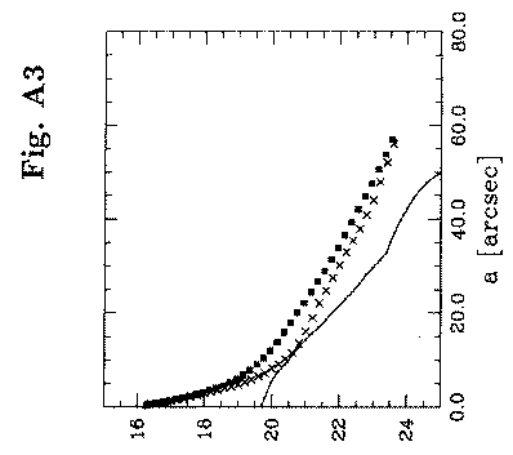

ก
5
6
0
0
0

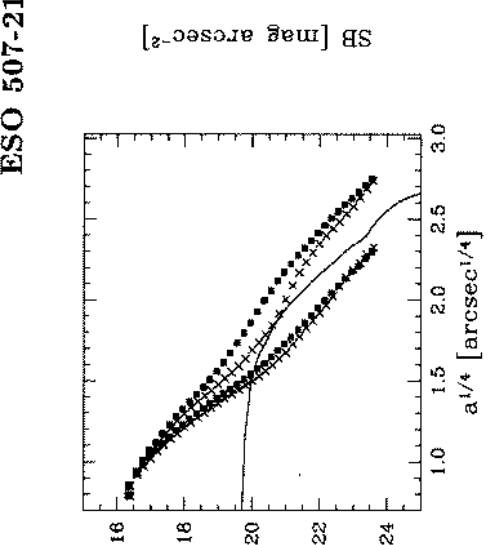

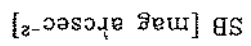

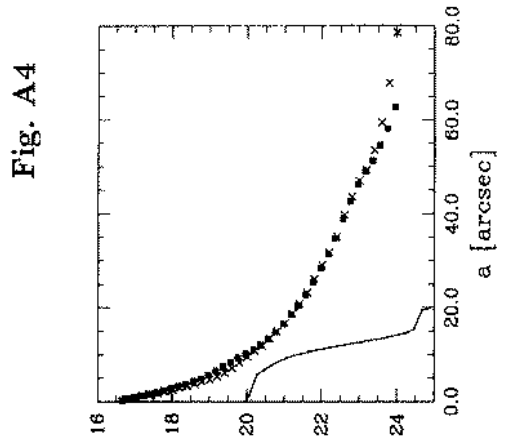

今ิ

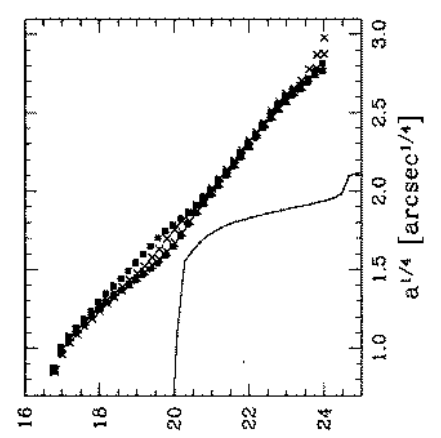

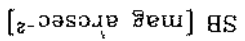

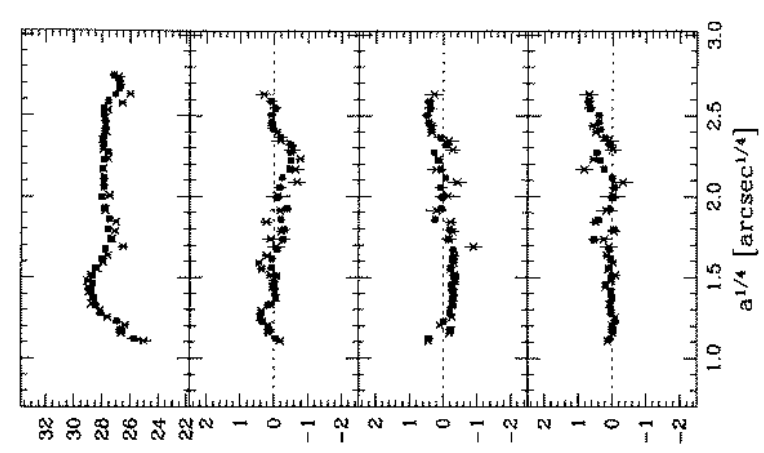

Vd $\quad 001 * \mathrm{e} /{ }^{\circ} \mathrm{q} \quad 00 \pi * \mathrm{e} / \mathrm{q} \quad 00 \pi^{*} / /^{\beta} \mathrm{q}$

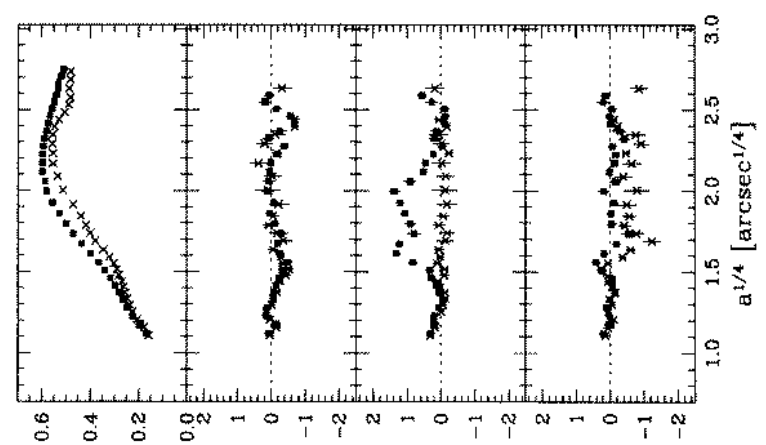

$B / q-1$

$00 T^{*} \mathrm{e} / \mathrm{se}$

$001 * \theta / \%$

$001 * 6 /{ }^{8} \mathrm{e}$

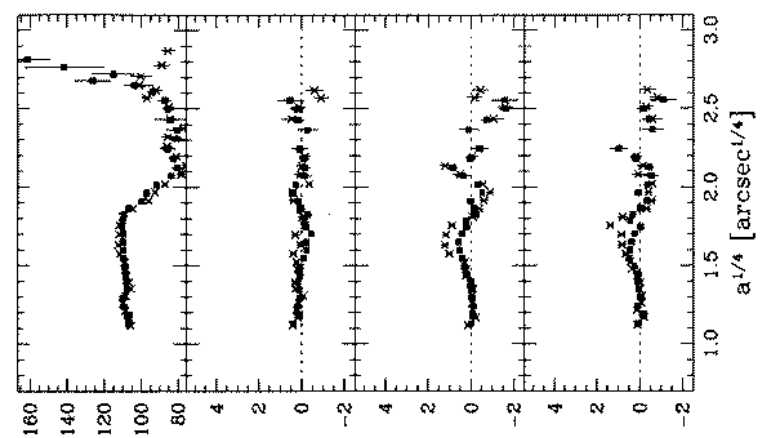

$\mathrm{Vd}$

$001 * \mathrm{e} /{ }^{\varepsilon} \mathrm{q} \quad 001 *^{\mathrm{e}} /{ }^{*} \mathrm{q} \quad 001{ }^{\mathrm{e}} /{ }^{8} \mathrm{q}$

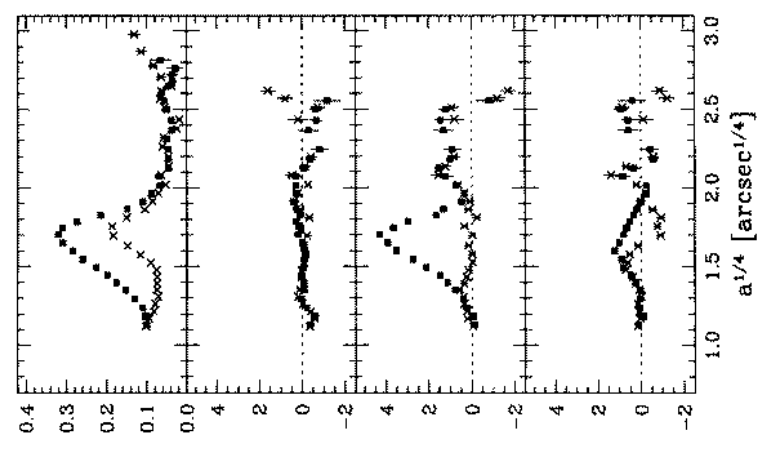

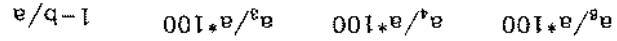




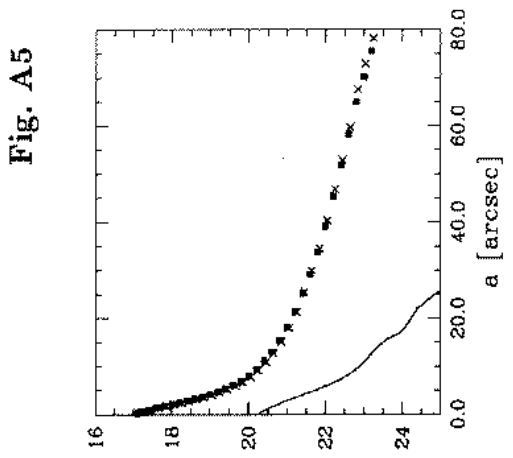

疋

U

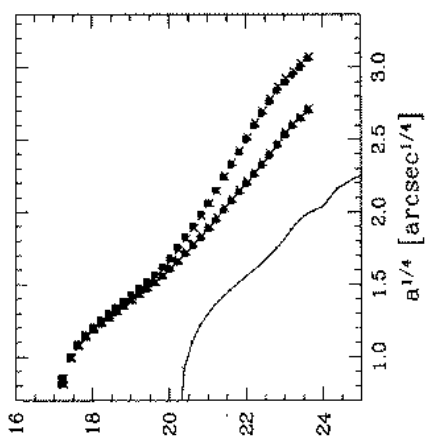

[z-pasare seur] gS

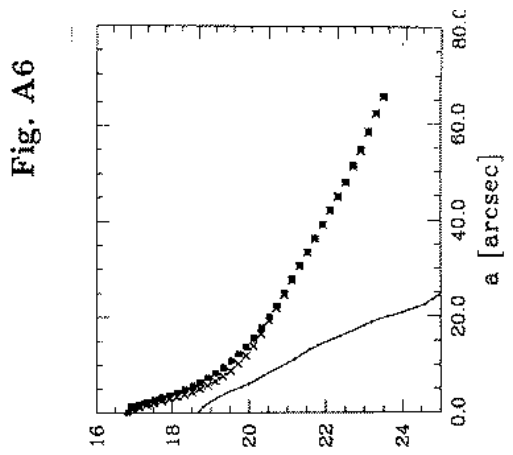

$\underset{\substack{\infty \\ \infty \\+}}{+\infty}$

$\underline{O}$

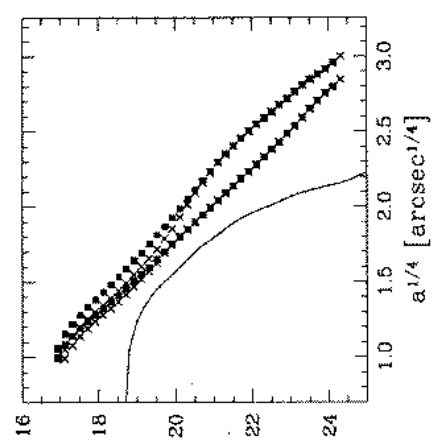

[z-oosore âeu $]$ gS

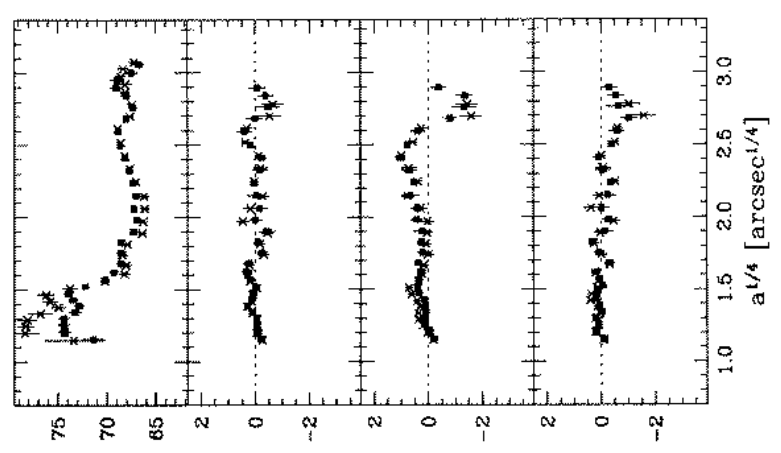

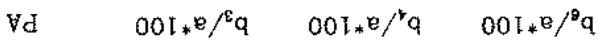

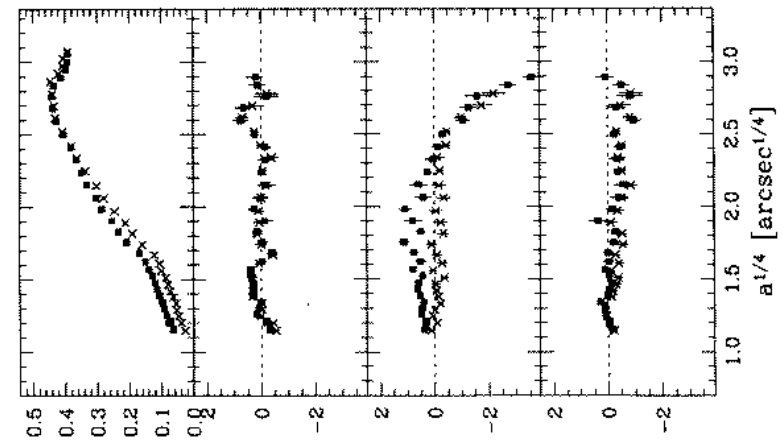

$e / q-1$

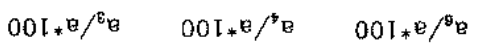

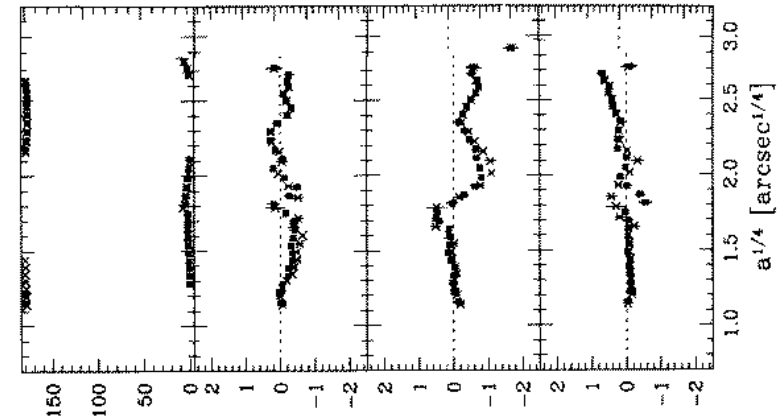

$\forall d \quad 001 * \mathrm{e} /{ }^{8} \mathrm{q} \quad 00 \% * \mathrm{e} / * \mathrm{q} \quad 001 * \mathrm{e} /{ }^{9} \mathrm{q}$

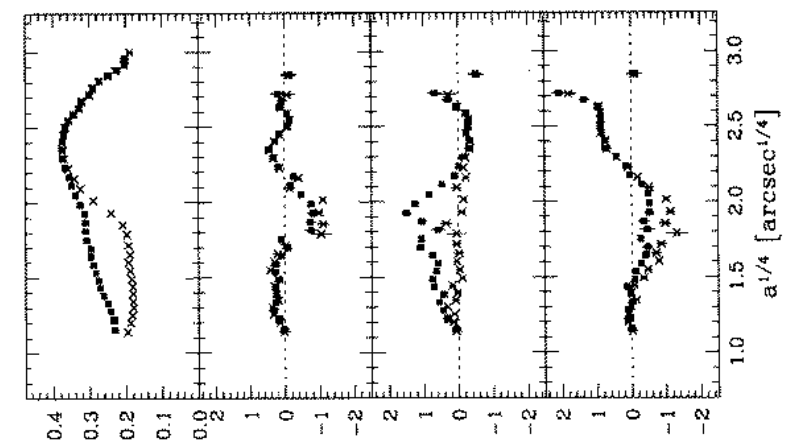

e/q-I

$001 *{ }^{B} / \mathrm{s}_{\mathrm{B}}$

$001 *^{e} /{ }^{*} \mathrm{e} \quad 001 *^{\mathrm{e}} /{ }^{8} \mathrm{e}$ 


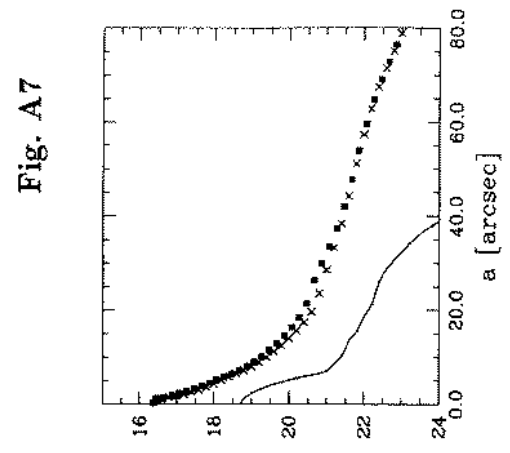

号
$ن$
$ن$
乙
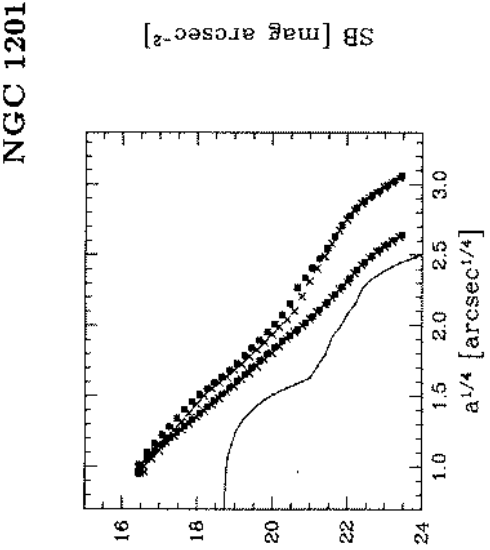

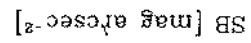

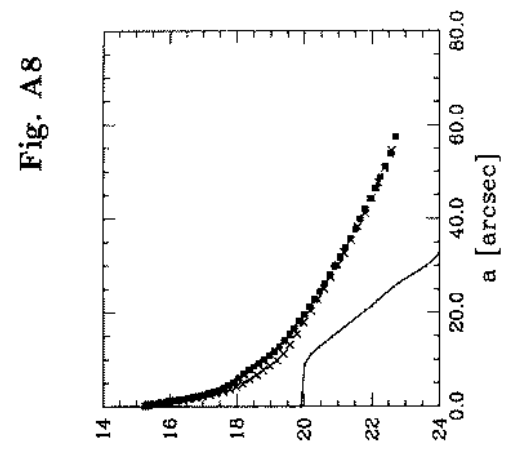

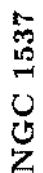

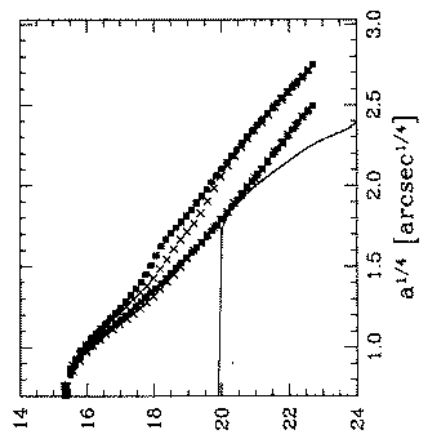

[z-oasone 8вu] gS

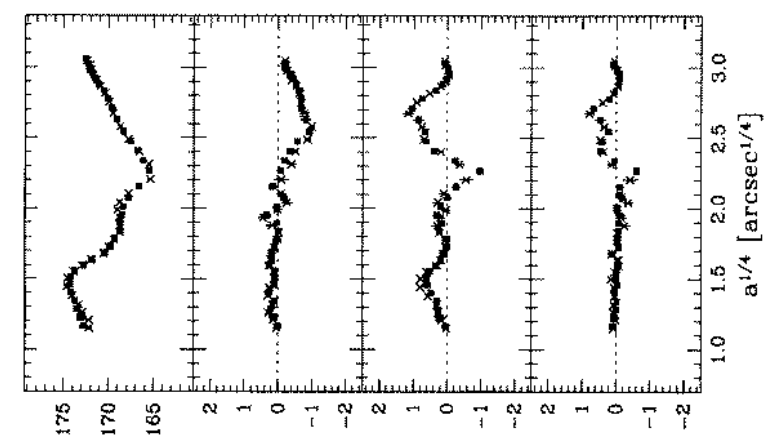

Vd $\quad 001 * \mathrm{e} /{ }^{\mathrm{g}} \mathrm{q} \quad 001 * \mathrm{~B} /{ }^{\mathrm{t}} \mathrm{q} \quad 001 * \mathrm{e} /{ }^{\circ} \mathrm{q}$

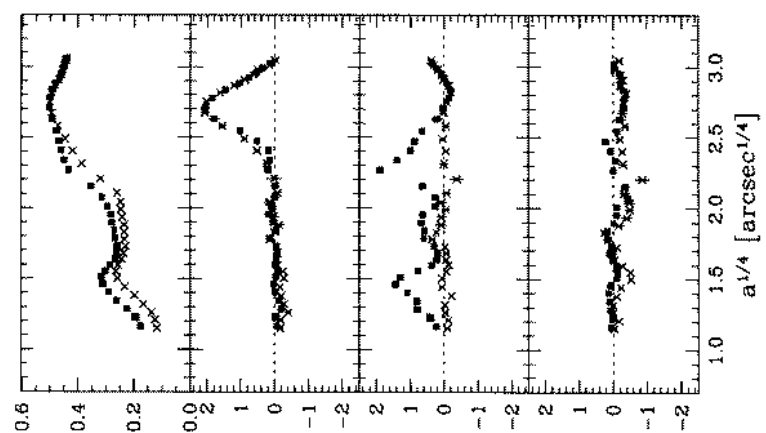

$e / q-I$

$001 * B / \varepsilon^{B}$

$001 * 8 /{ }^{*} \mathrm{~B}$

$00 \mathrm{I} * \mathrm{P} /{ }^{\mathrm{B}} \mathrm{e}$

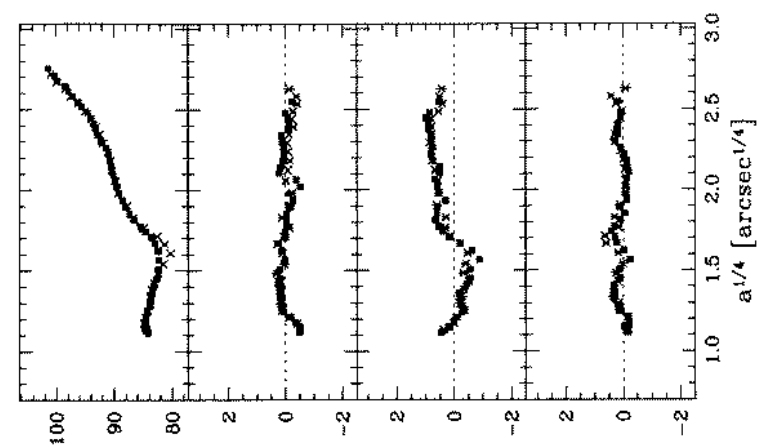

Vd

$00{ }^{*} \mathrm{e} /{ }^{\varepsilon} \mathrm{q} \quad 00 \mathrm{I} * \mathrm{e} /{ }^{*} \mathrm{q} \quad 00 \mathrm{~T} * \mathrm{e} /{ }^{*} \mathrm{q}$

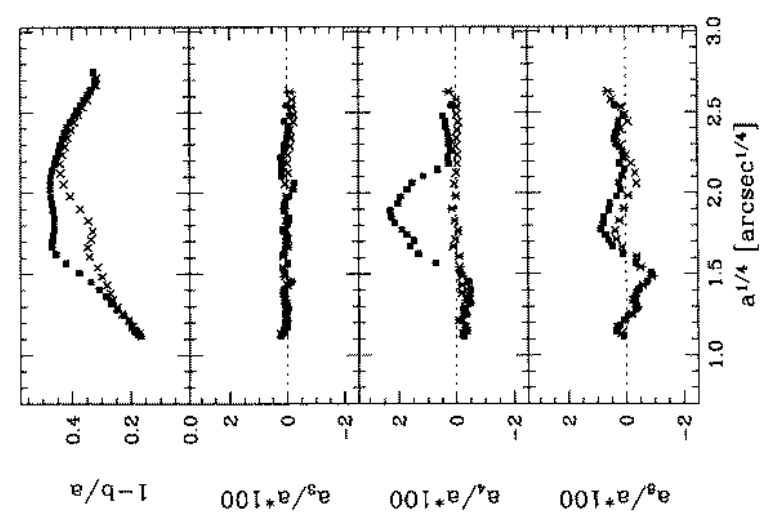



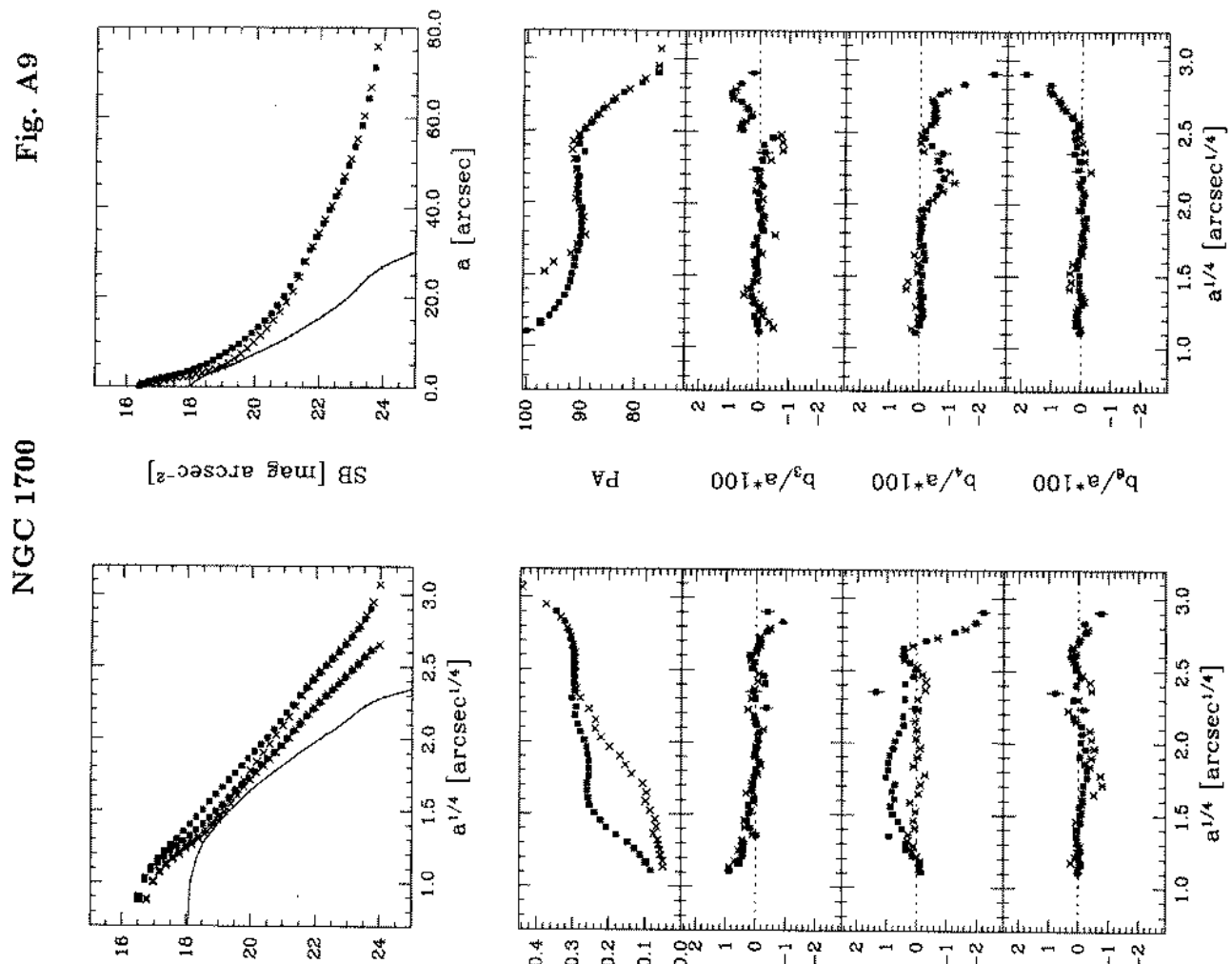

Vd $\quad 001 * 8 /{ }^{\varepsilon} \mathrm{q} \quad 001 * \mathrm{e} /{ }^{*} \mathrm{q} \quad 001 * \mathrm{e} /{ }^{\circ} \mathrm{q}$

[z-จวSวนB รeu]

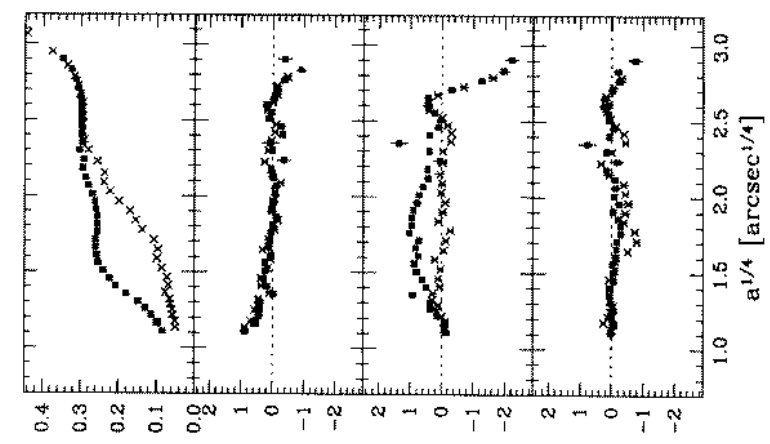

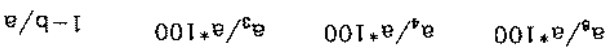
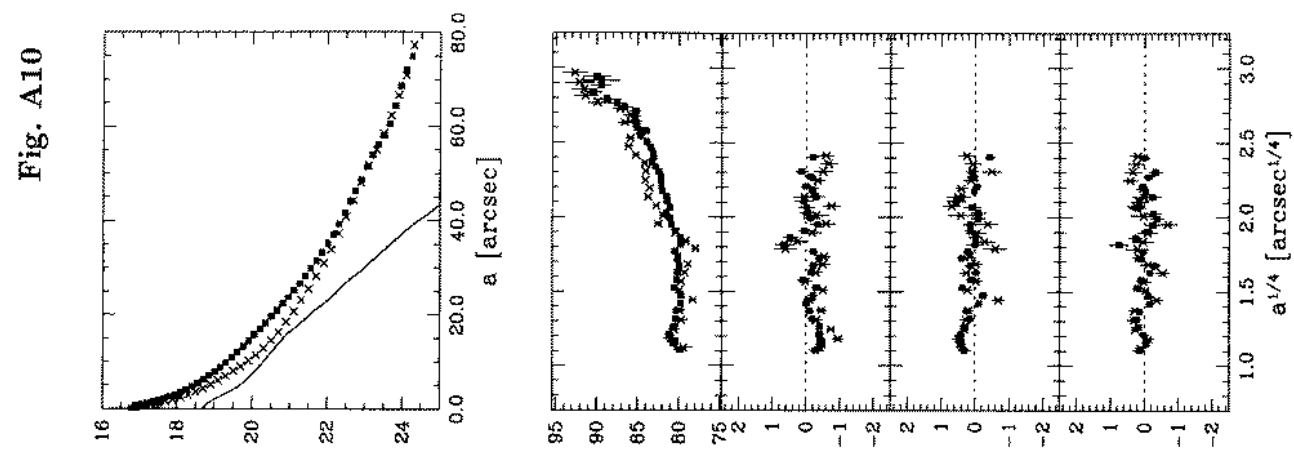

N
N
U
$Z$

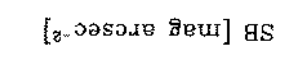

$\forall d$

$001 * e /{ }^{\circ} \mathrm{q}$

$001 * \mathrm{e} / \mathrm{t} \mathrm{q}$

$001 * \mathrm{e} /{ }^{9} \mathrm{q}$

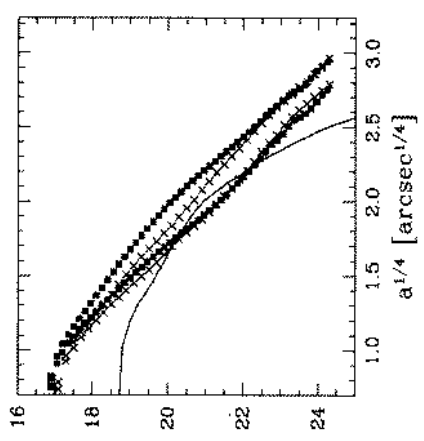

[z-วasose รew] gS

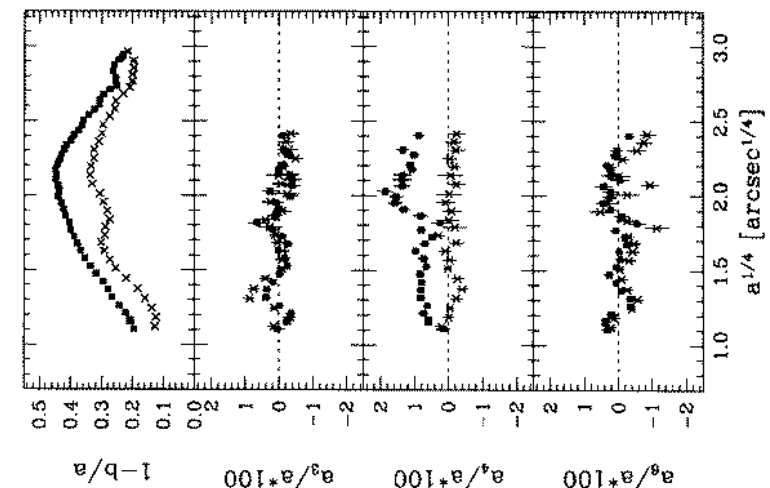




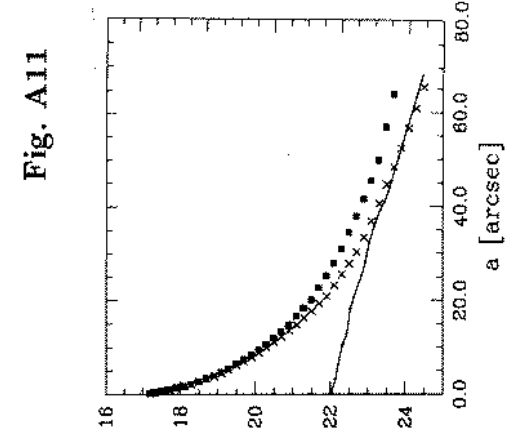

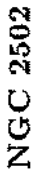

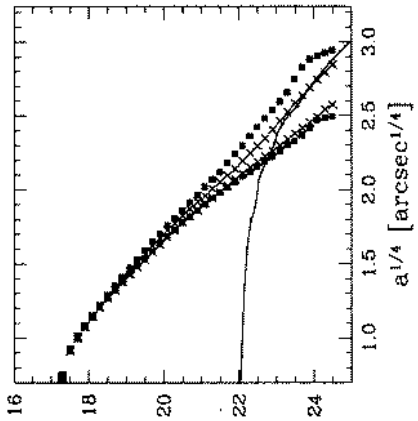

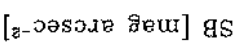

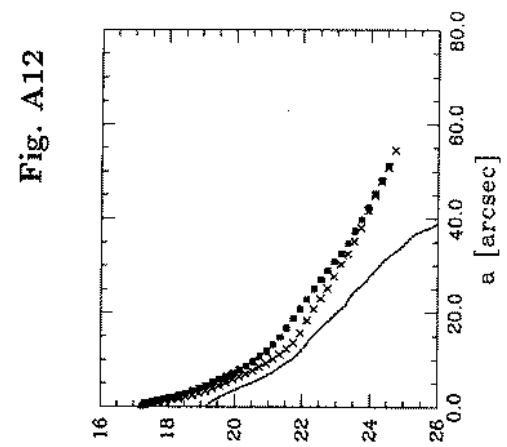

$\stackrel{5}{8}$
0
0
$Z$

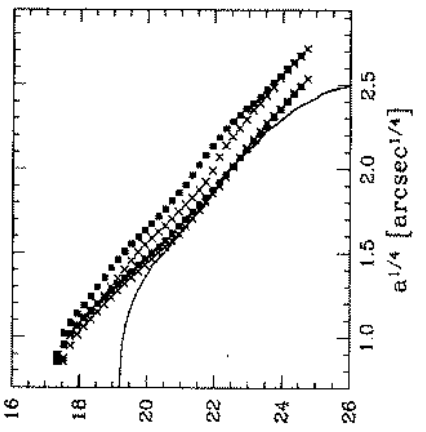

[z-oasore geu $]$ as

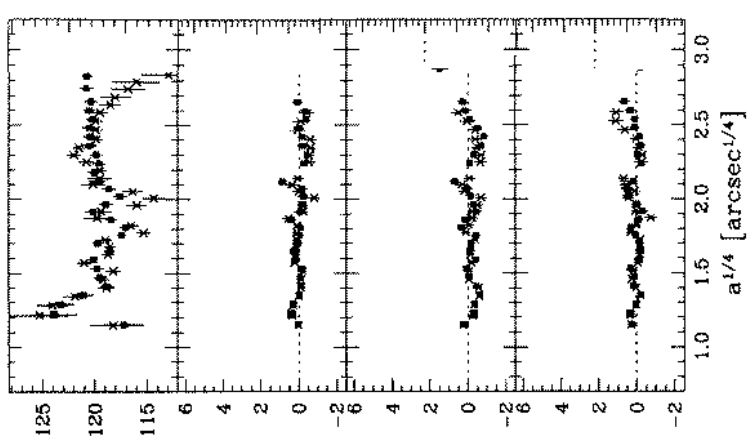

$\forall d$

$001 * e / e^{\varepsilon} q$

$00 r_{*}^{e} / \mathrm{t} q$

$001 * 8 /{ }^{\circ} \mathrm{q}$

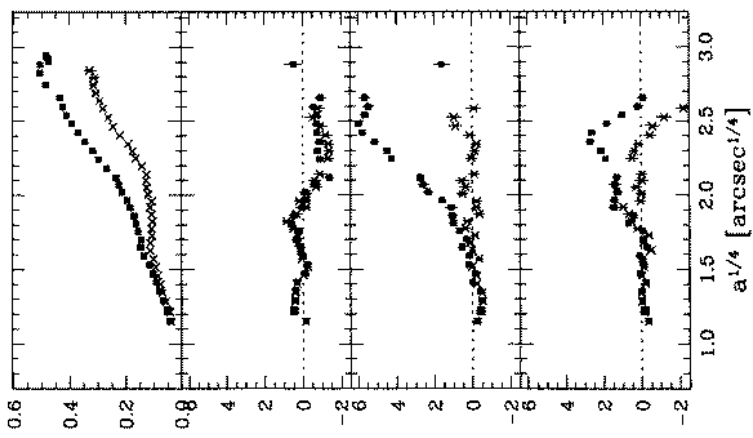

$e / q-I$

$001 * 8 /{ }^{8} 8$

$00 I_{*} \mathrm{e} / \mathrm{s} \mathrm{B}$

$001 *^{e} /{ }^{8} \mathrm{~B}$

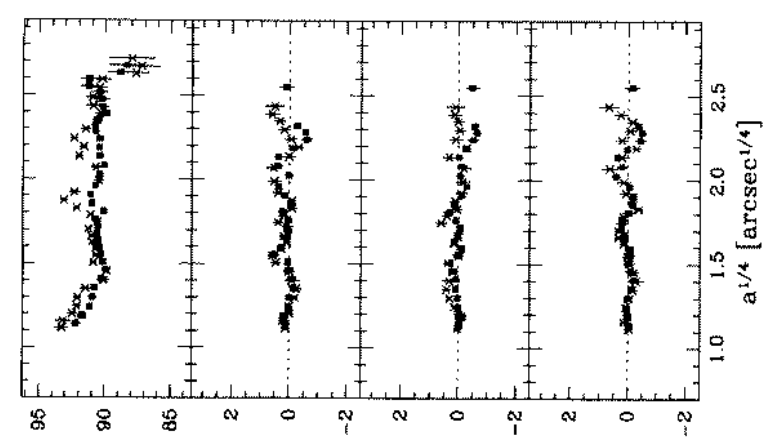

$\mathrm{Vd}$

$001 * \mathrm{e} / \mathrm{e}^{\mathrm{q}} \mathrm{q} \quad 001 * \mathrm{e} /{ }^{*} \mathrm{q} \quad 001 * \mathrm{e} /{ }^{\mathrm{p}} \mathrm{q}$

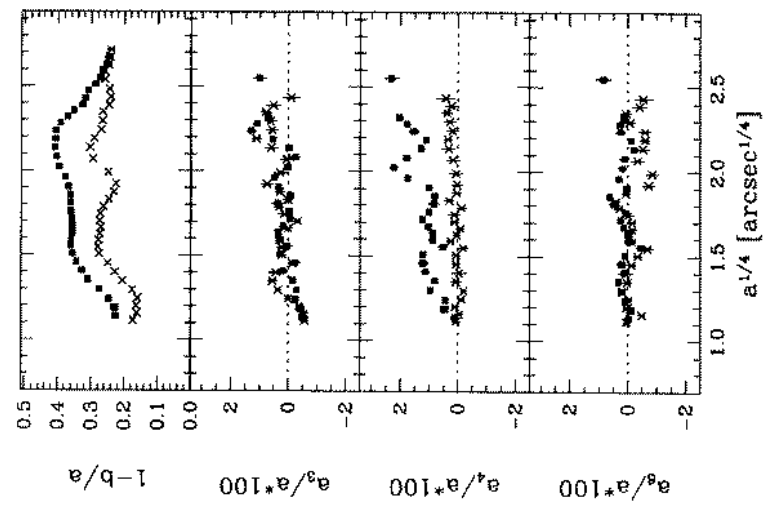




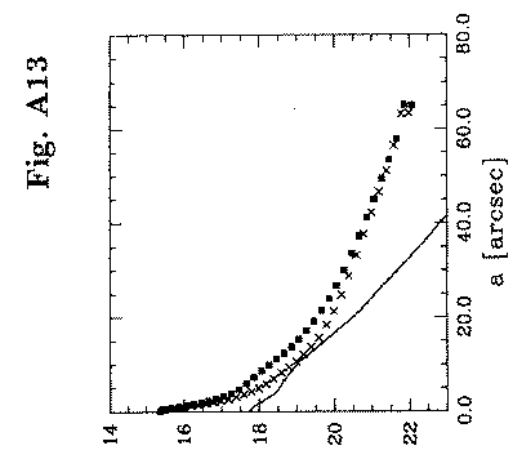

10
$\infty$
10
10 [z-oasode geur] gS

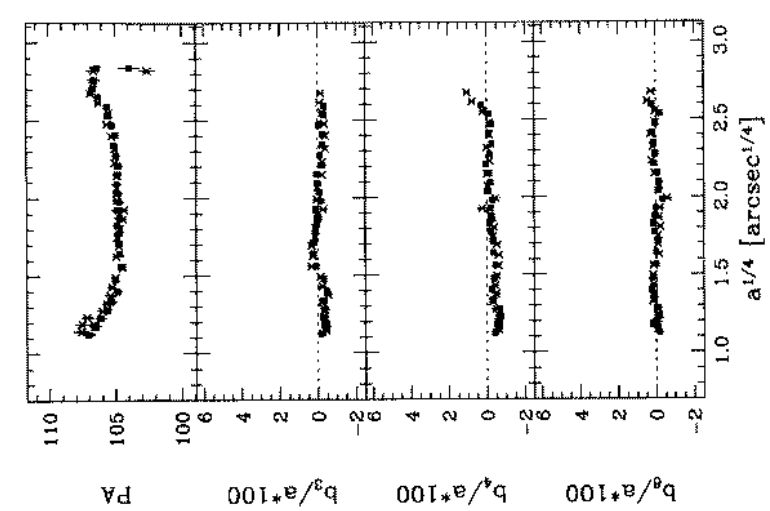

导
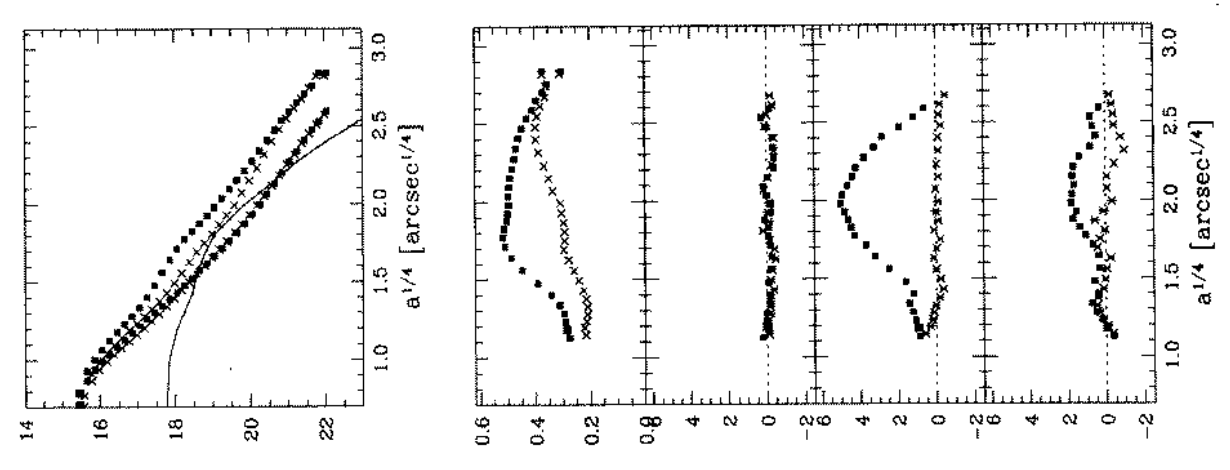

[z-วaspุเ 8ียน] $]$ gS

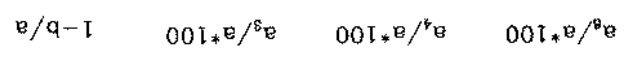
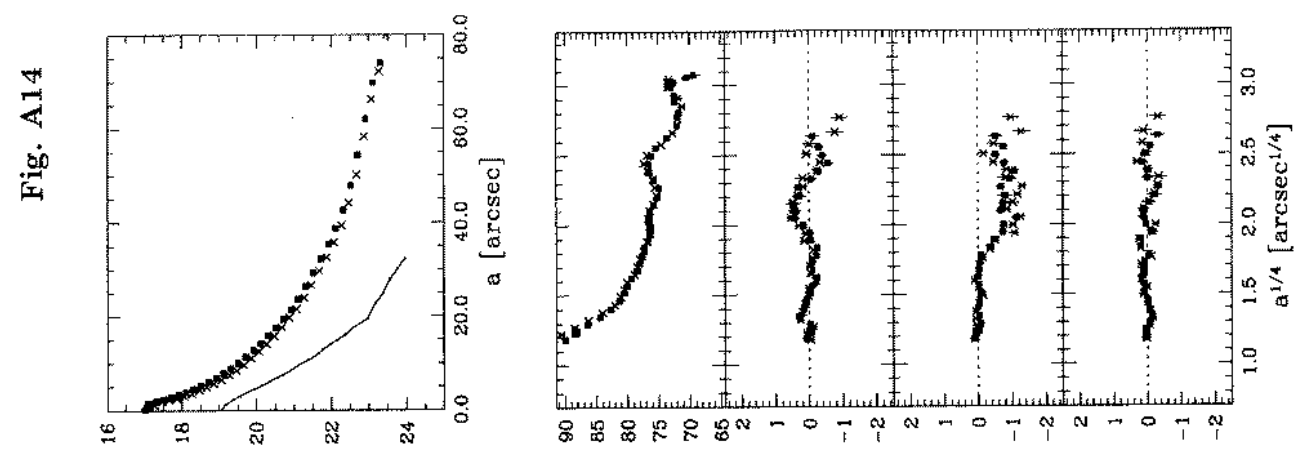

0
0
0
0
$Z$
$Z$

[z-oesore gem] gS

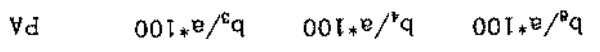
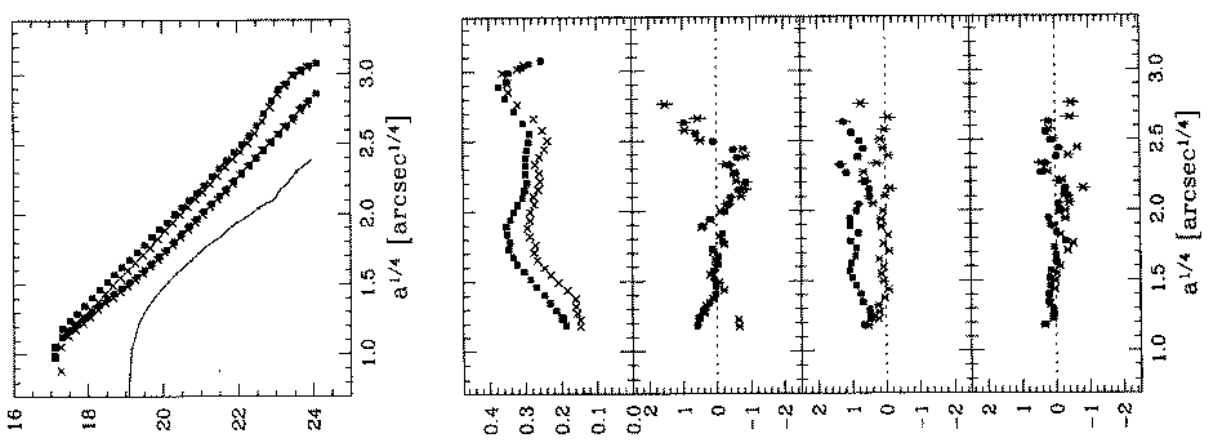

[z-oasode gew] as

B/q-I

$001 * \mathrm{e} / \mathrm{s}^{\mathrm{e}}$

$001 * \mathrm{e} /{ }^{\mathrm{e}} \mathrm{e}$

$001 * \mathrm{e} / \mathrm{e}$ 


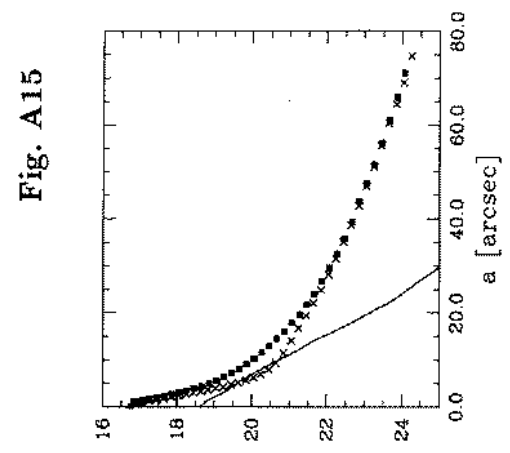

$\infty$
$\stackrel{\infty}{\infty}$
0
0
0
$Z$
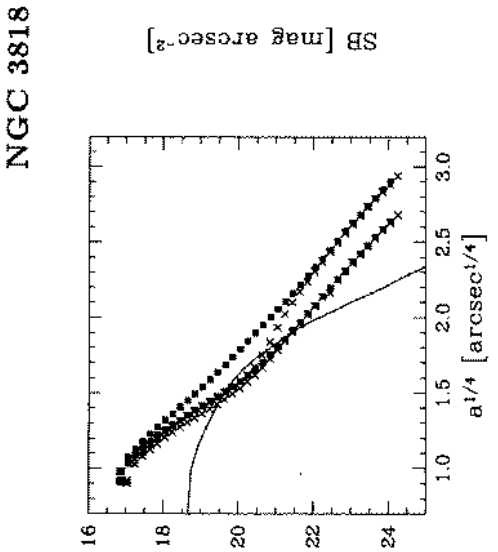

[z-osone gem] ds

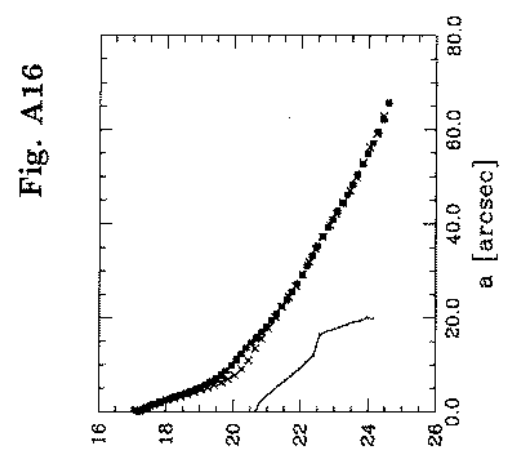

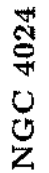

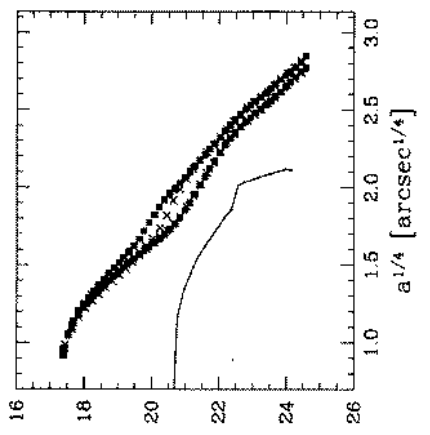

[z-วasวre geur] des

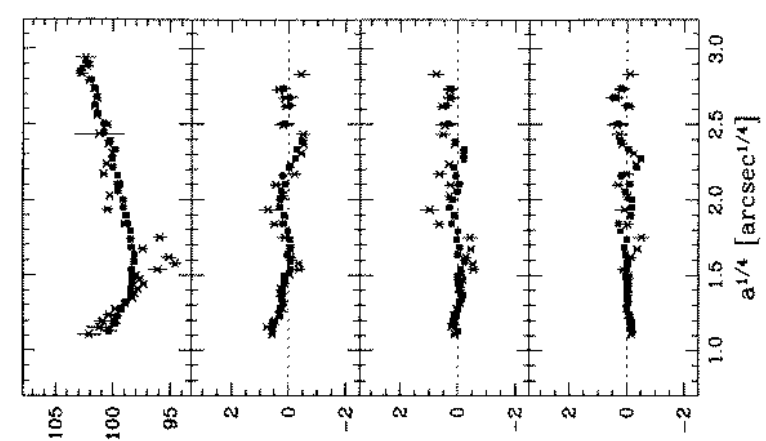

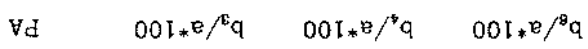

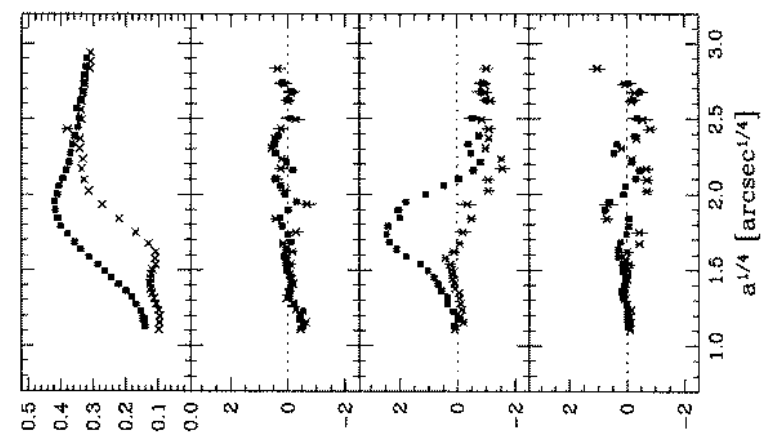

$e / q-I$

$001 * 0 /{ }^{8} \mathrm{e}$

$00 t * \mathrm{e} /{ }^{*} \mathrm{e}$

$001 *^{\mathrm{B} /{ }^{\circ} \mathrm{e}}$

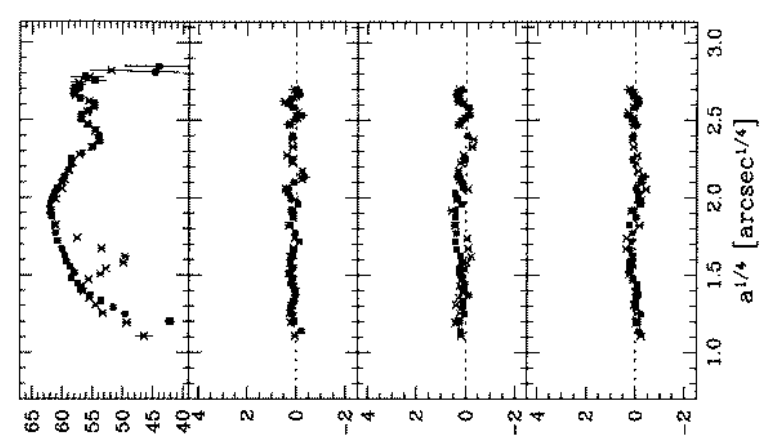

Yd

$001 * \mathrm{e} / \mathrm{e}^{\mathrm{e}}$

$00 \mathrm{I} * \mathrm{e} / \mathrm{pq}$

$00{ }_{*} \mathrm{e} /{ }^{\circ} \mathrm{q}$

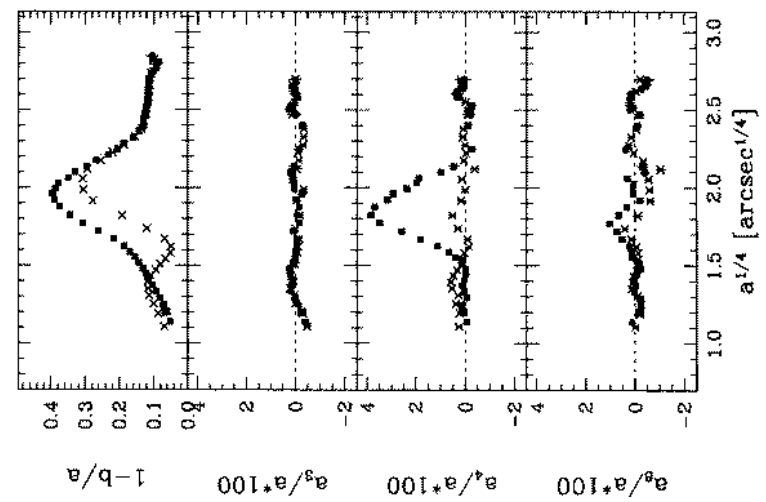



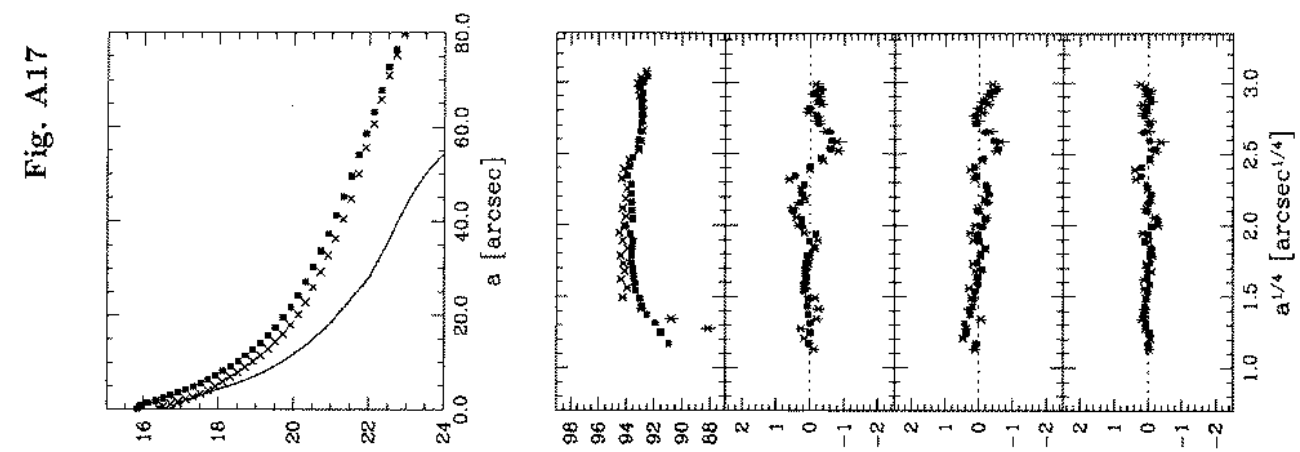

0
4
0
4
$Z$

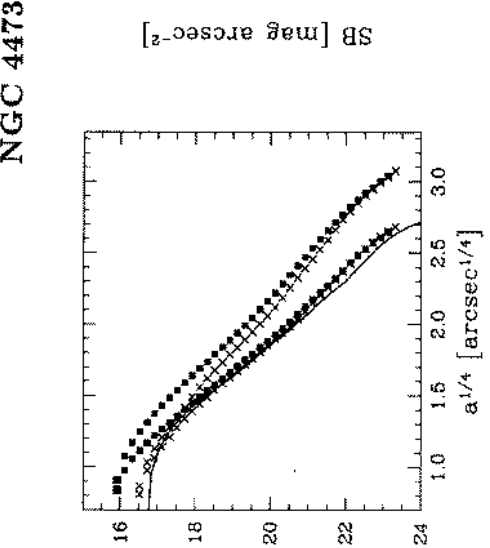

$\forall d$

$001 * \mathrm{~B} /{ }^{\circ} \mathrm{q} \quad 001 * \mathrm{E} /{ }^{\mathrm{p}} \mathrm{q} \quad 001 * \mathrm{E} /{ }^{\mathrm{a} q}$

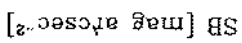

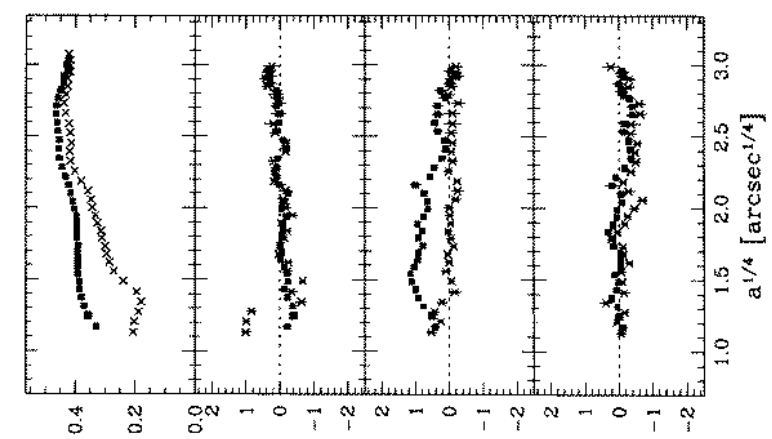

e/q- I

$001 * 0 / s^{8}$

$001 * \mathrm{~B} /{ }^{*} \mathrm{~B}$

$001 * \mathrm{E} /{ }^{8} \mathrm{e}$
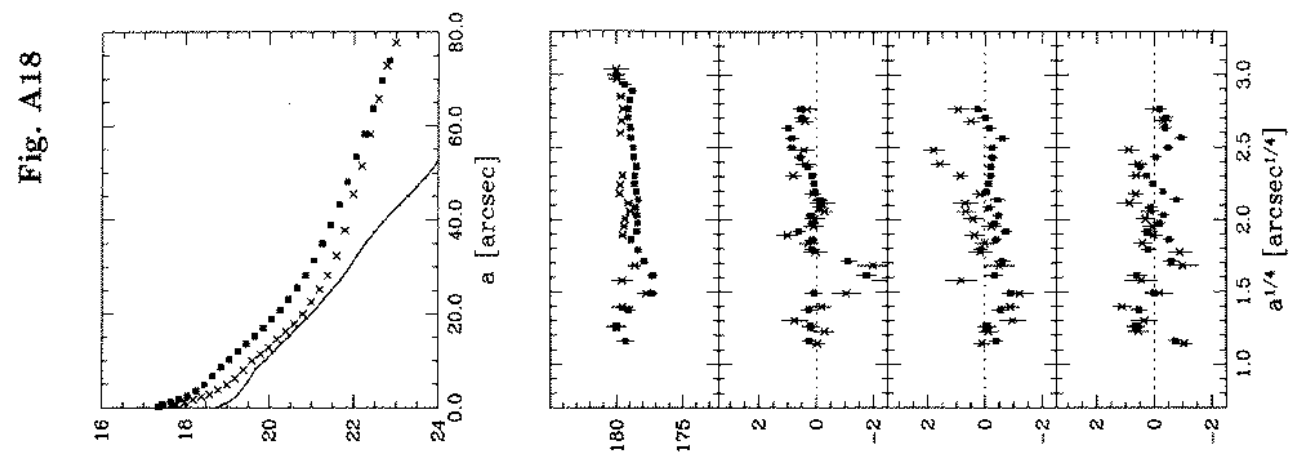

0
10
0
0
0
7

[z-Dasode aew] gS

$\forall d$

$001 *^{\mathrm{e} /{ }^{\mathrm{s}} \mathrm{q}} \quad 001 * \mathrm{e} /{ }^{\mathrm{p}} \mathrm{q} \quad 001 * \mathrm{~B} /{ }^{\circ} \mathrm{q}$
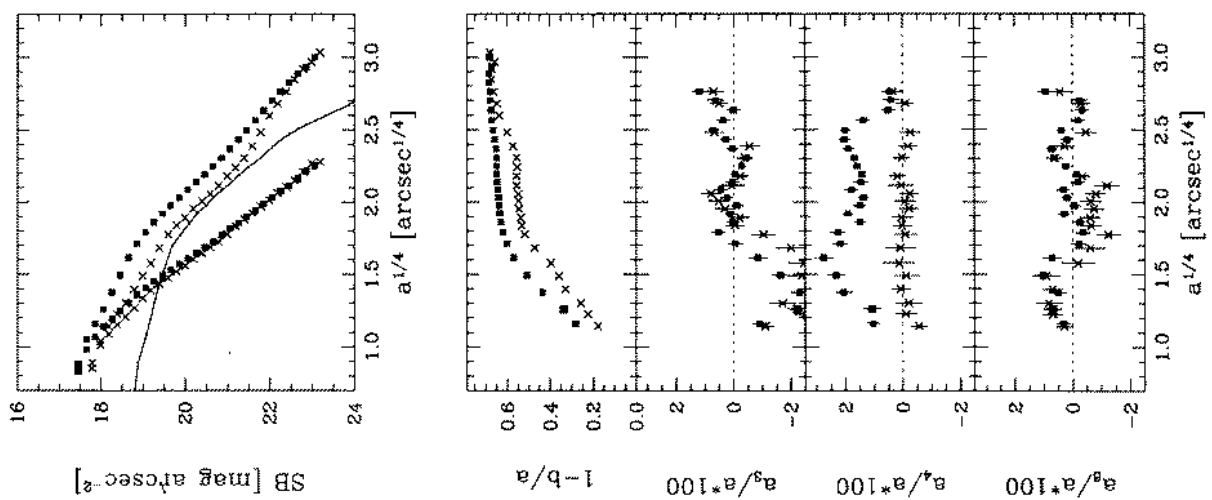

[z-oasote genu] aS

e/q-1

$001 * \mathrm{~B} / \mathrm{s}_{\mathrm{B}}$

$001 \times 8 / 0$

$001 * 9 / 9$ 


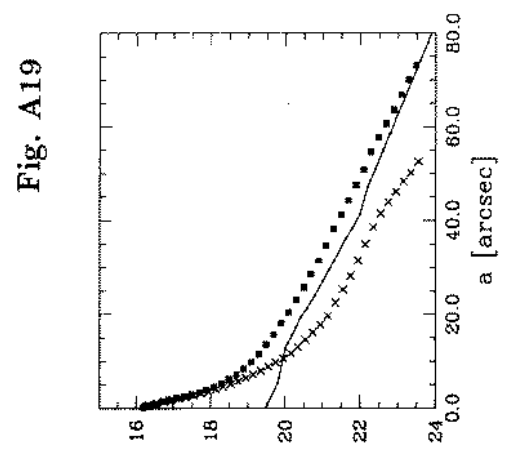

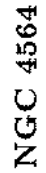
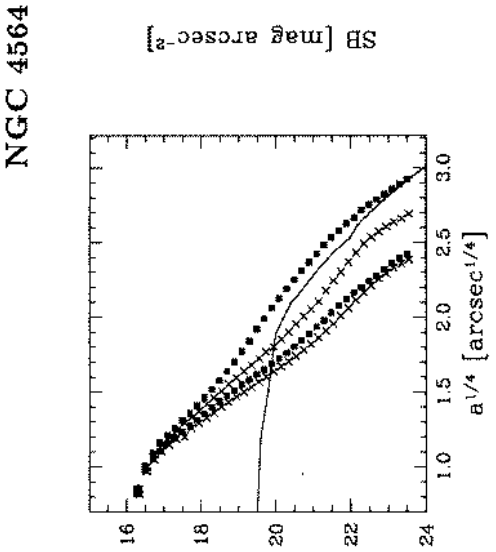

[a-oasoste 8eut] de

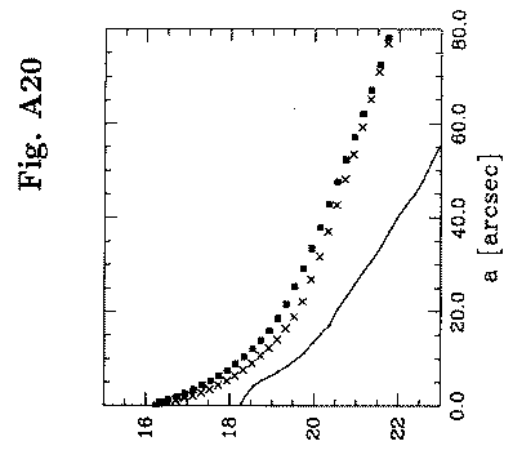

5
0
0
$己$
$Z$

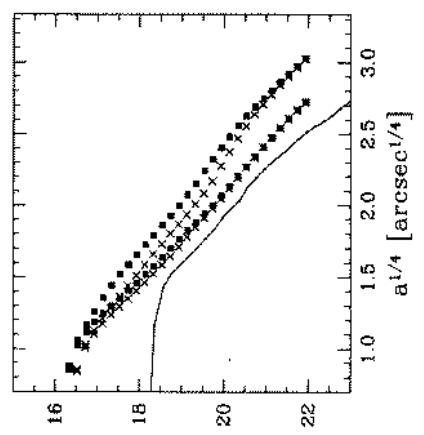

[z-oasare gent] aS

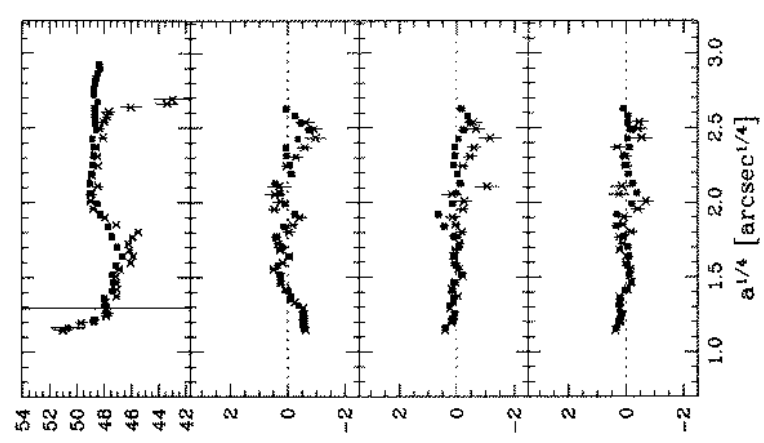

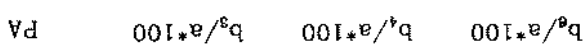

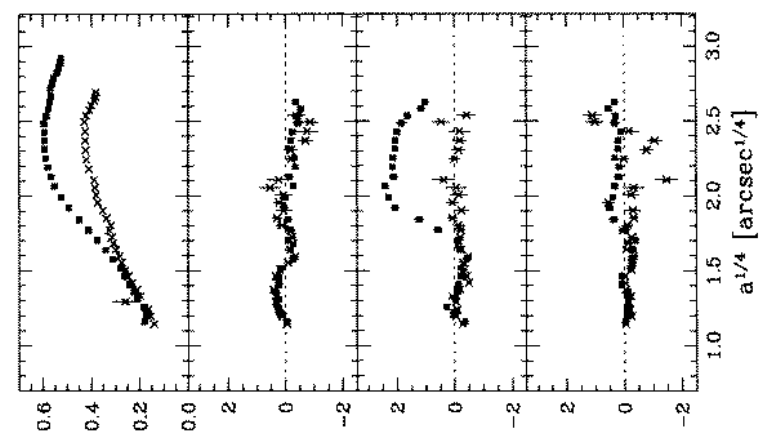

$e / q-1$

$00 \tau_{*} \mathrm{e} / \mathrm{\varepsilon}_{\mathrm{B}}$

$00 *_{*} B /{ }^{*} \mathrm{E}$

$001 * \mathrm{e} /{ }^{\mathrm{B}} \mathrm{g}$

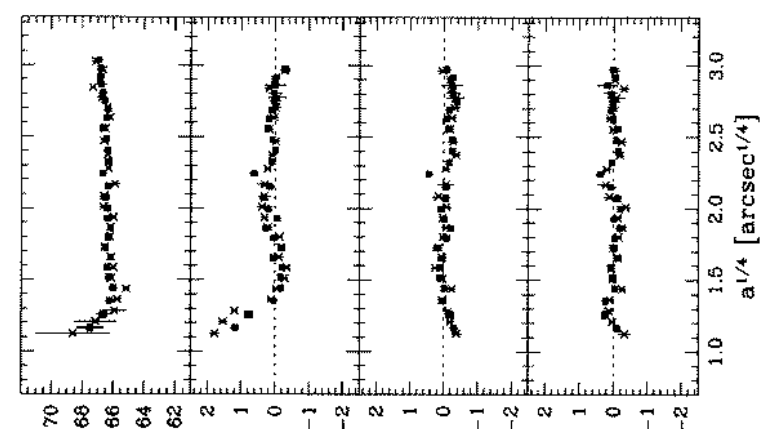

Vd

$001 * e /{ }^{e} \mathrm{q}$

$00 \mathrm{~T} * \mathrm{e} / \mathrm{q}$

$00 r_{*} \mathrm{e} /{ }^{\circ} \mathrm{q}$

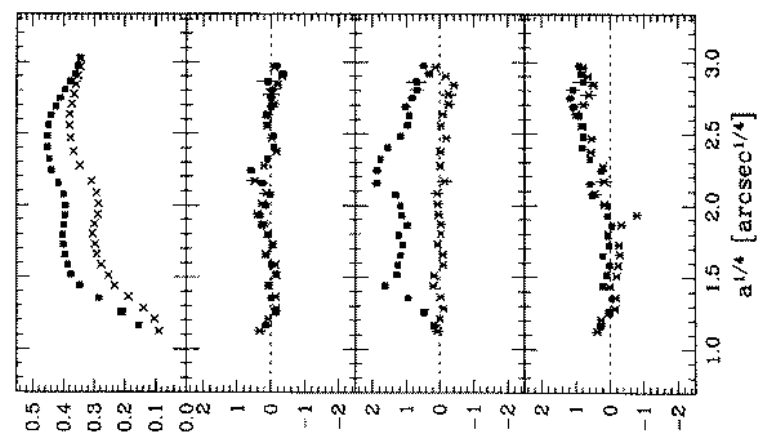

e/q-I

$001 * 8 /{ }^{\varepsilon} \mathrm{e}$

$001 * \mathrm{e} / \mathrm{p} \mathrm{B}$

$001 * \mathrm{e} /{ }^{\mathrm{e} e}$ 


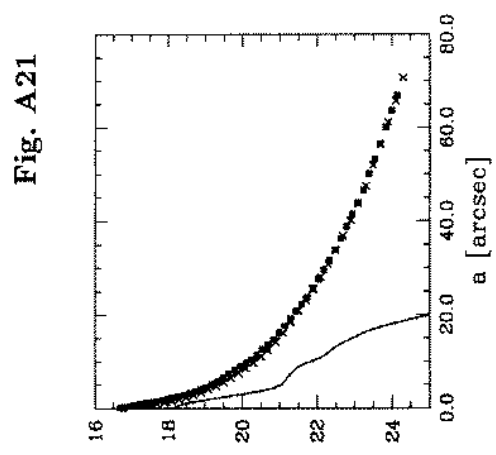

0
0
0
0
$z$
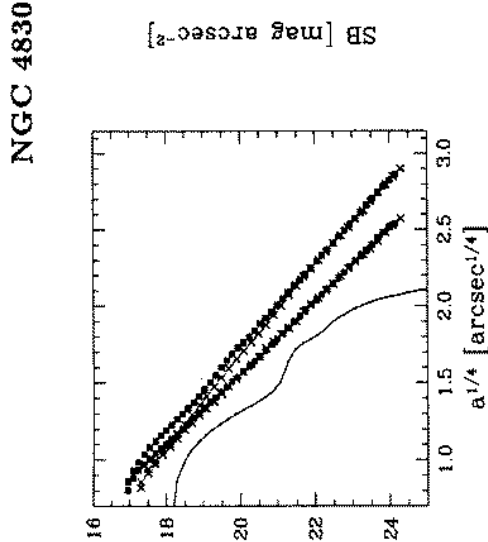

[z-כasore geur] as

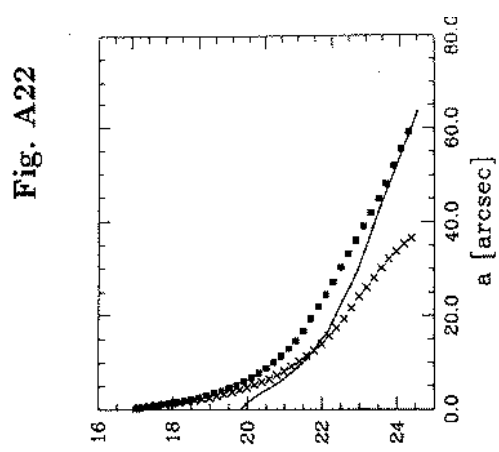

\begin{tabular}{l}
$\infty$ \\
0 \\
0 \\
0 \\
\hdashline
\end{tabular}

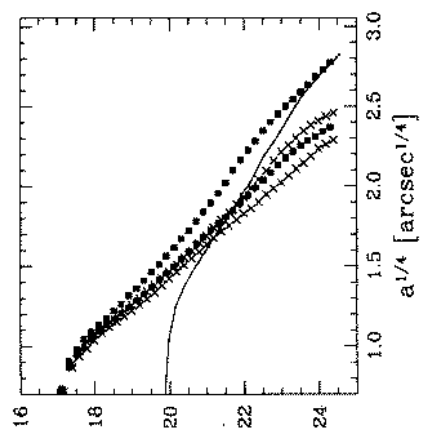

[z-วosว.x gew] gS

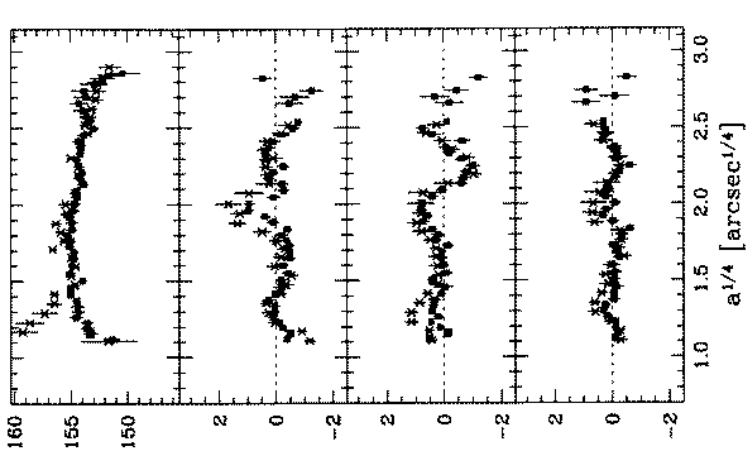

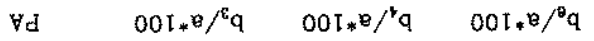
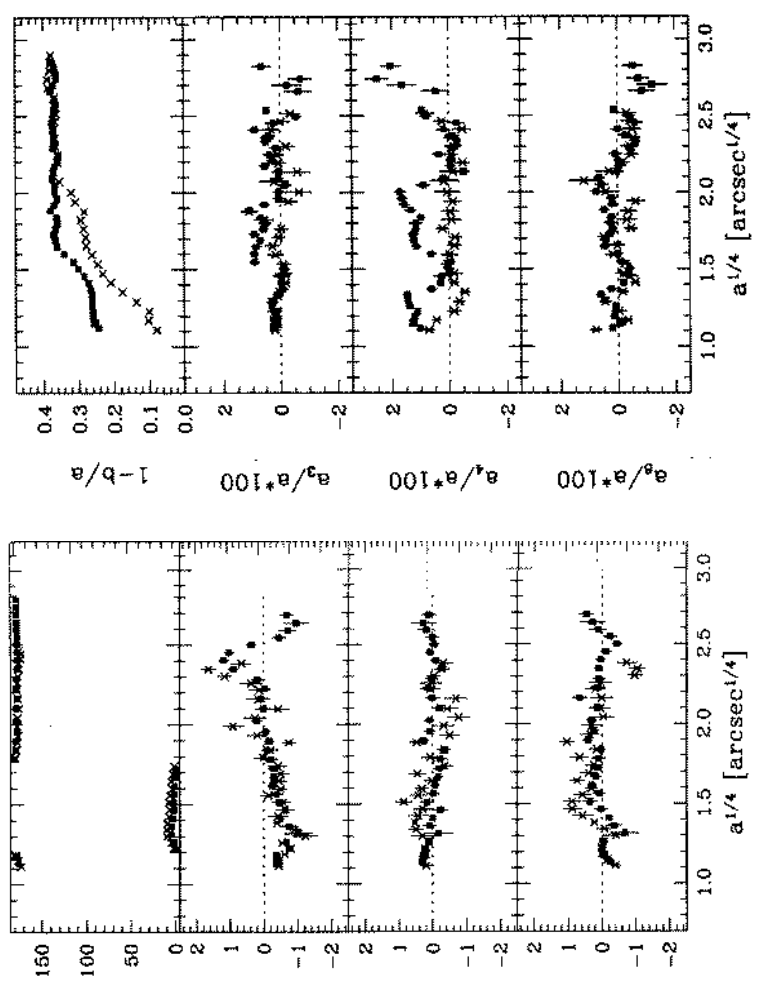

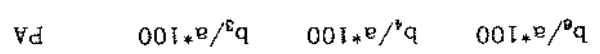

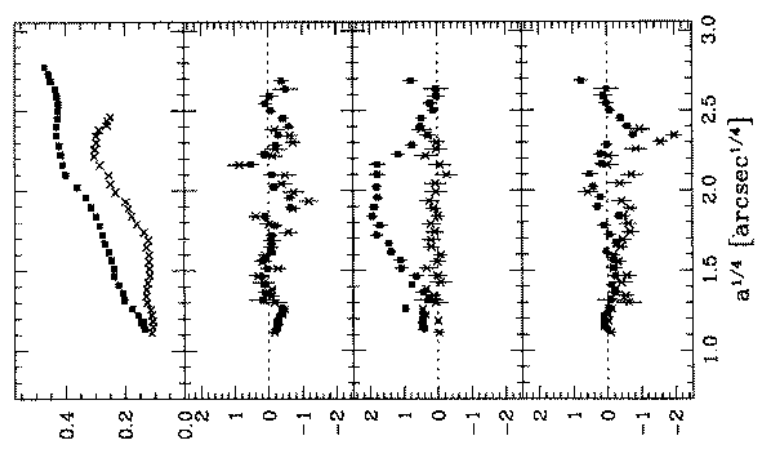

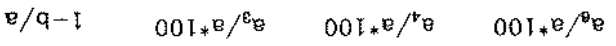




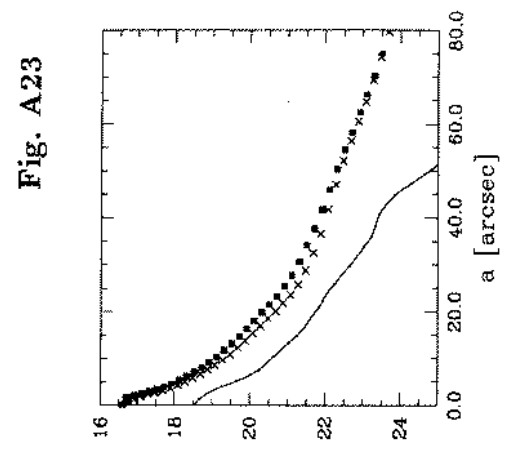

$\infty$
0
0
0
$Z$
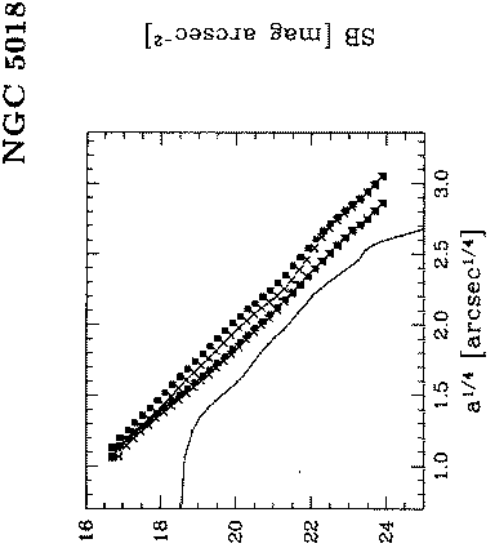

[z-วasofiด gew] gS

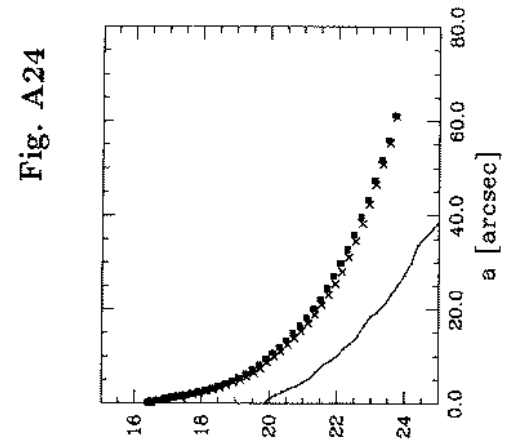

$\infty$
1
0
0
0
0
$Z$

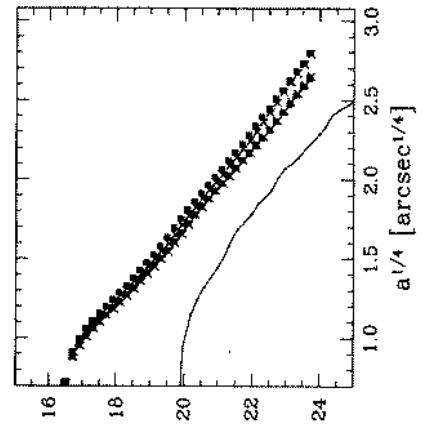

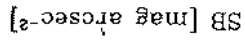

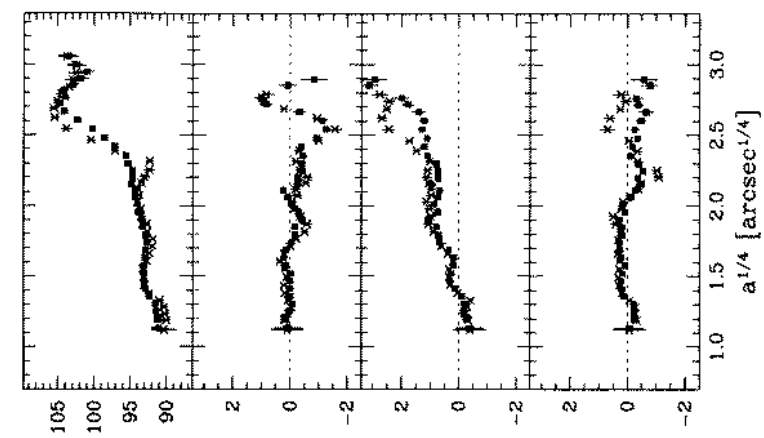

$\forall d \quad 001 * \mathrm{e} / \mathrm{s} \mathrm{q} \quad 001 * \mathrm{e} /{ }^{*} \mathrm{q} \quad 001 *{ }^{\mathrm{e} /{ }^{\circ} \mathrm{q}}$

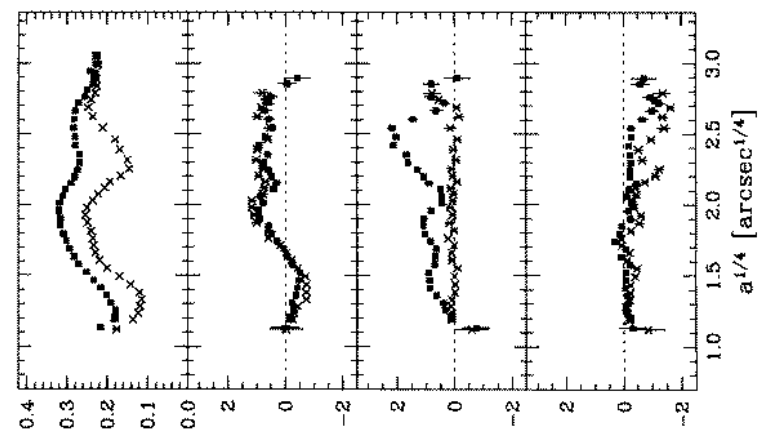

e/q-I

$001 *^{0} /{ }^{8} \mathrm{e}$

$0,1 * 6 / p^{3}$

$001 * \mathrm{e} /{ }^{8} \mathrm{e}$

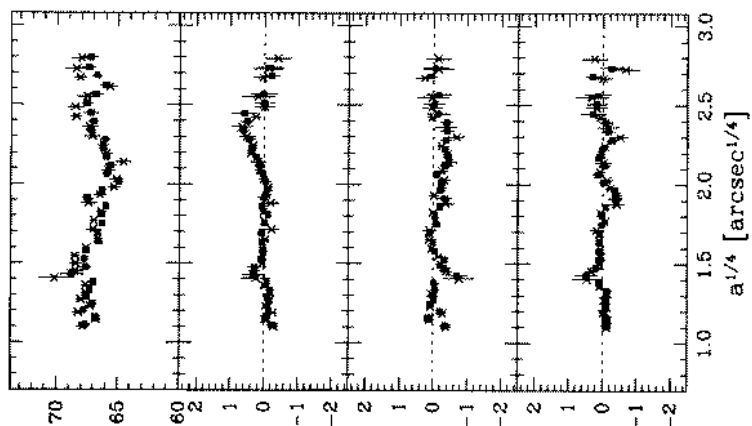

$\forall d$

$00 \mathrm{~L} * \mathrm{e} /{ }^{\mathrm{s}} \mathrm{q} \quad 001 * \mathrm{e} /{ }^{*} \mathrm{q}$

$001 * 2 /{ }^{\circ} \mathrm{q}$

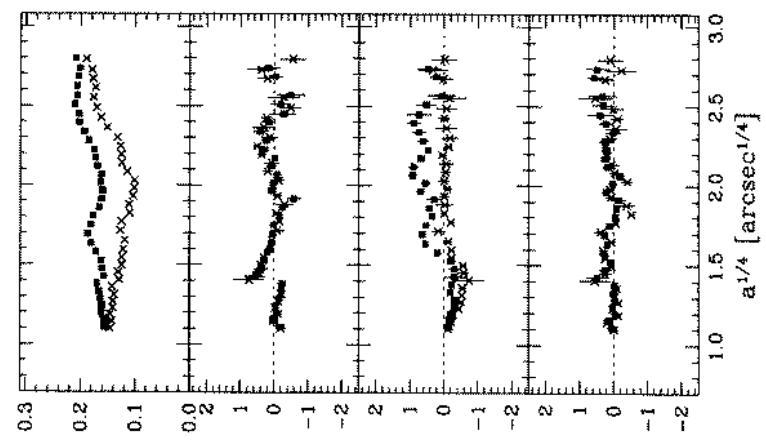

$8 / q-1$

$001 * B /{ }^{\mathrm{e} e}$

$001 *^{B} /{ }^{p} \mathrm{~B}$

$001 * \mathrm{e} /{ }^{8} \mathrm{e}$ 


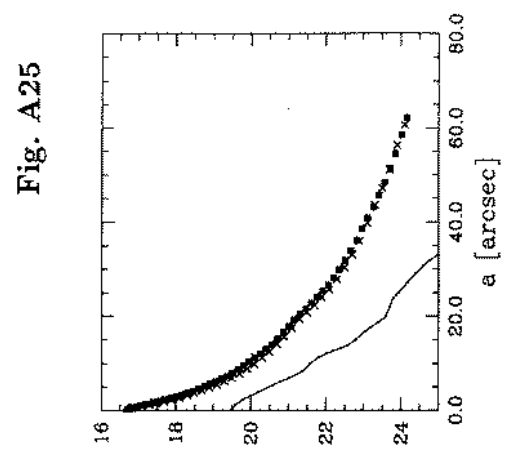

$\overrightarrow{10}$
0
0
0
0
$Z$

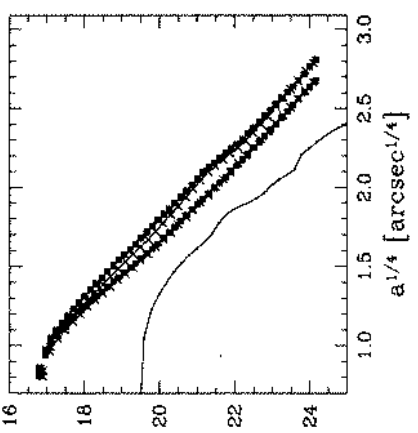

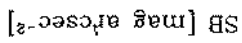

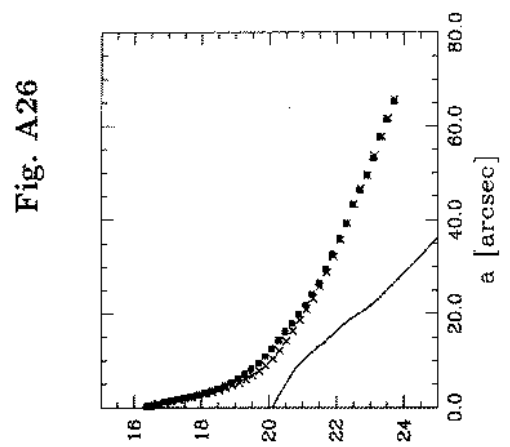

ญิ

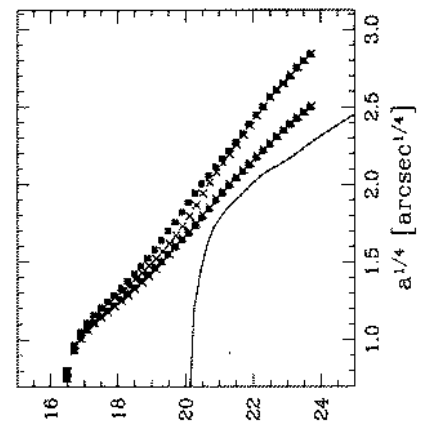

[z-oasou gew] gs

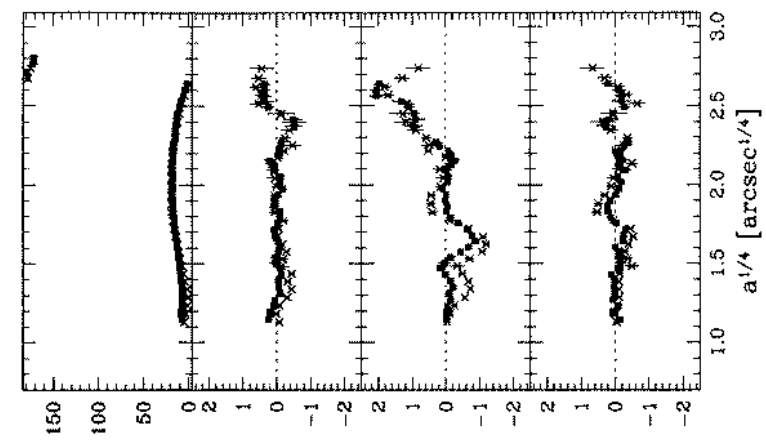

$\forall \mathrm{d} \quad 00 I * \mathrm{~B} /{ }^{\mathrm{e}} \mathrm{q} \quad 00 \mathrm{I} * \mathrm{~B} /{ }^{\circ} \mathrm{q} \quad 001 * 8 /{ }^{8} \mathrm{q}$

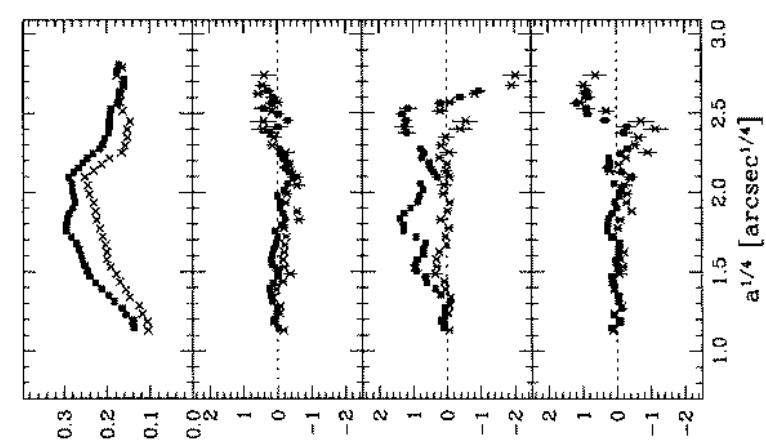

$B / q-$

$00 \pi_{*} \mathrm{~B} / \mathrm{s}_{\mathrm{B}}$

$00 l_{*} \mathrm{e} /{ }^{\mathrm{B}} \mathrm{B}$

$001 * e /{ }^{\beta} \mathrm{e}$

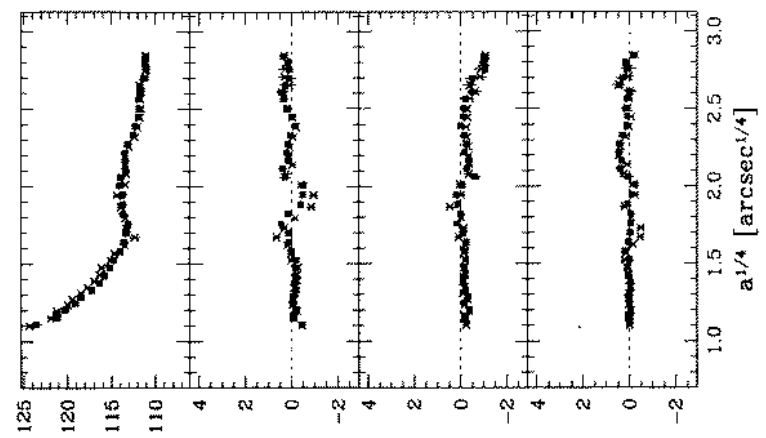

Vd $\quad 001 * \mathrm{e} /{ }^{\mathrm{e}} \mathrm{q} \quad 001 * \mathrm{e} / \mathrm{q} \quad 001 * \mathrm{e} /{ }^{\circ} \mathrm{q}$

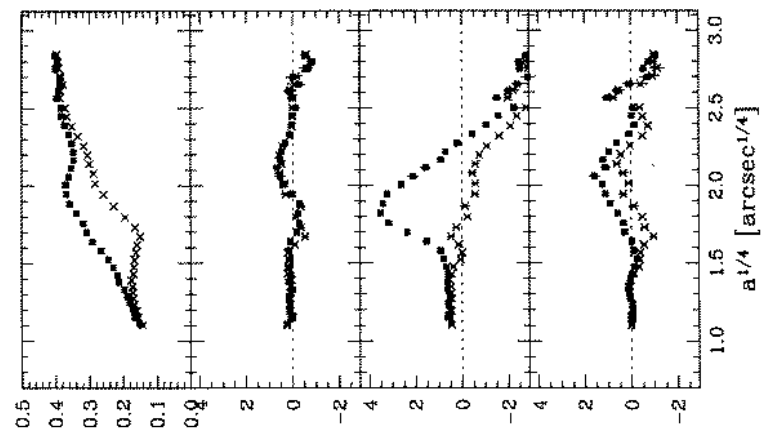

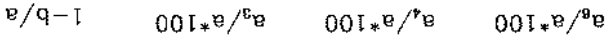




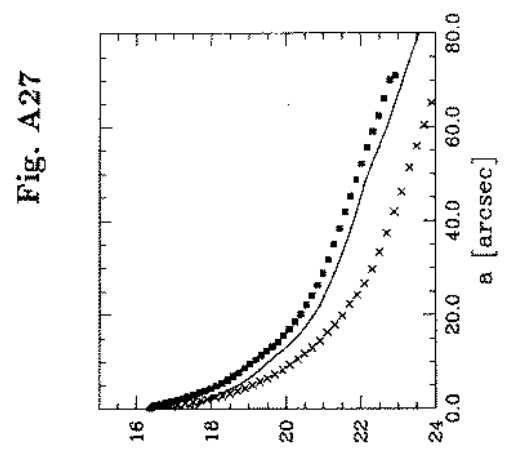

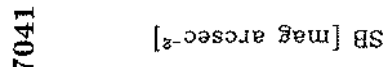
U

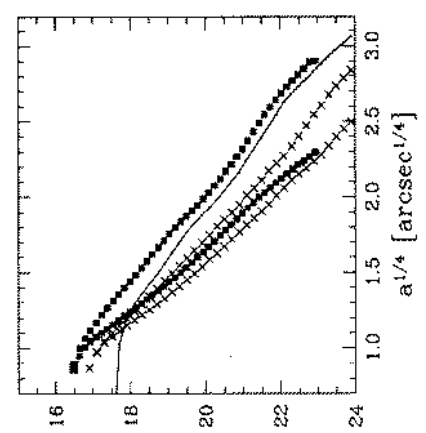

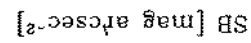

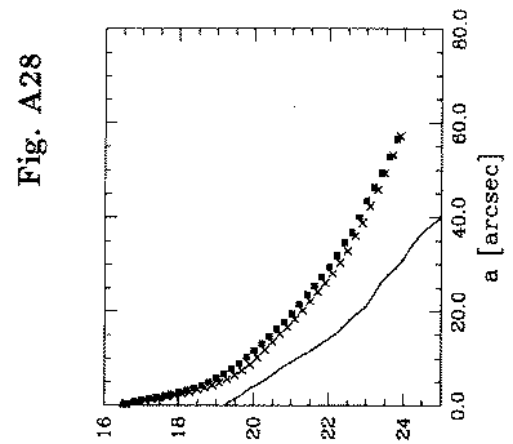

5
0
0
0
$Z$

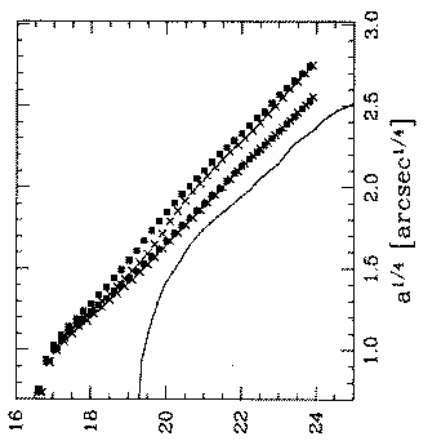

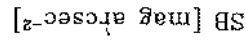

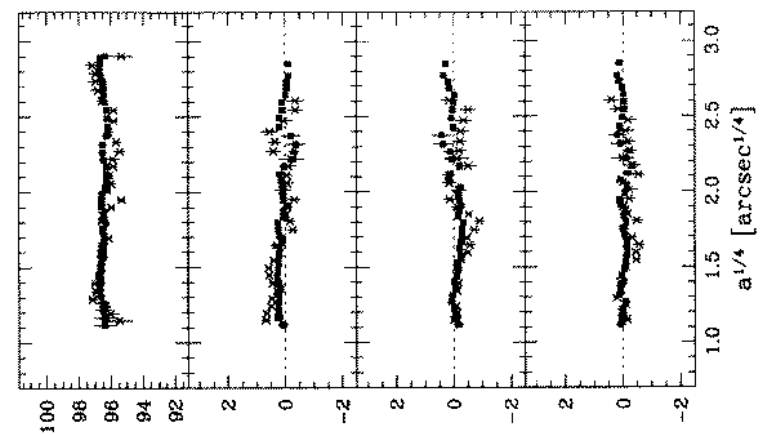

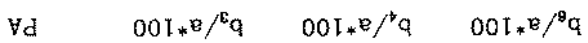

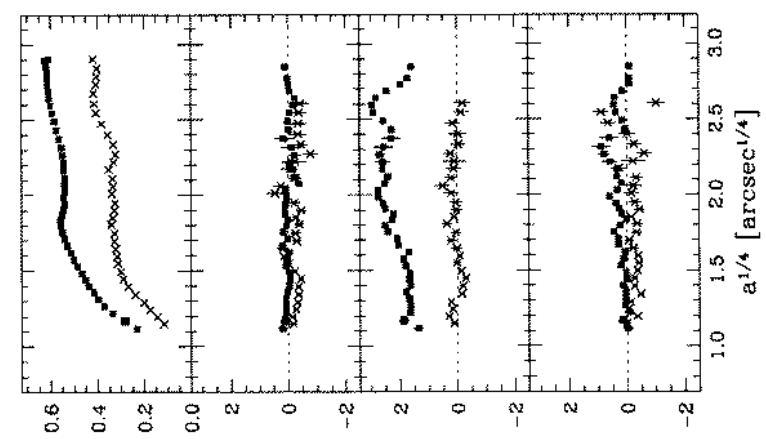

e/q-i

$001 * \mathrm{e} / \mathrm{se}$

$001 * \mathrm{e} / \mathrm{pe}$

$001 * \mathrm{~B} /{ }^{\circ} \mathrm{e}$

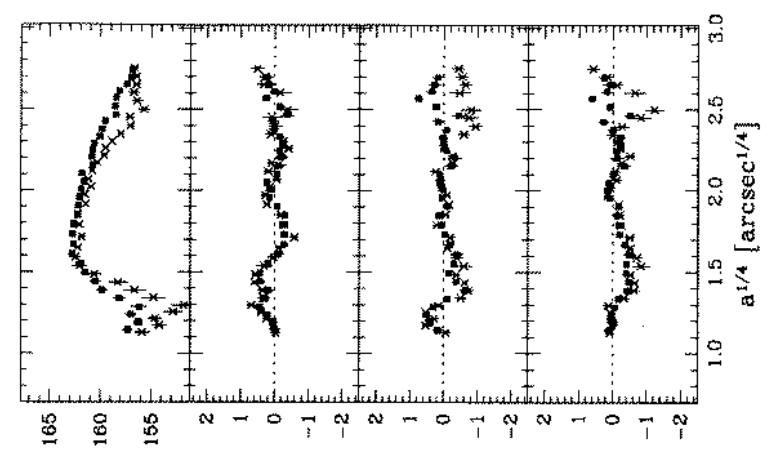

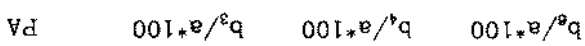

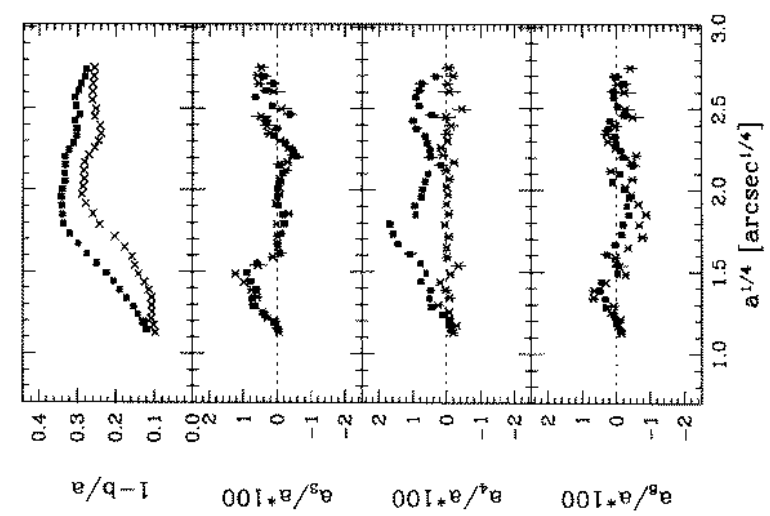

